\title{
Design Considerations for MEMS-Scale Piezoelectric Mechanical Vibration Energy Harvesters
}

\author{
Noël E. duToit, ${ }^{1}$ Brian L. Wardle, ${ }^{1, *}$ and Sang-Gook Kim² \\ ${ }^{1}$ Department of Aeronautics and Astronautics, Massachusetts Institute of Technology, \\ 77 Massachusetts Avenue, 33-314 Cambridge, MA 02139, USA \\ ${ }^{2}$ Department of Mechanical Engineering, Massachusetts Institute of Technology, \\ 77 Massachusetts Avenue, 33-314 Cambridge, MA 02139, USA
}

\begin{abstract}
Design considerations for piezoelectric-based energy harvesters for MEMS-scale sensors are presented, including a review of past work. Harvested ambient vibration energy can satisfy power needs of advanced MEMS-scale autonomous sensors for numerous applications, e.g., structural health monitoring. Coupled 1-D and modal (beam structure) electromechanical models are presented to predict performance, especially power, from measured low-level ambient vibration sources. Models are validated by comparison to prior published results and tests of a MEMS-scale device. A non-optimized prototype low-level ambient MEMS harvester producing $30 \mu \mathrm{W} / \mathrm{cm}^{3}$ is designed and modeled. A MEMS fabrication process for the prototype device is presented based on past work.
\end{abstract}

Keywords: Energy scavenging; power harvesting; MEMS; piezoelectric; vibration energy conversion; wireless sensors

\section{INTRODUCTION}

In recent years the development of distributed wireless sensor node networks has been a focus of several research groups. Research projects include SenseIt from DARPA, SmartDust at UC Berkeley [1], $\mu$-AMPS at MIT [2], and i-Bean wireless transmitters from Millennial Net, Inc. [3]. Distributed wireless microsensor networks have been described as systems of ubiquitous, low-cost, selforganizing agents (or nodes) that work in a collaborative manner to solve

Received November 26, 2004; In final form February 4, 2005.

*Corresponding author. E-mail: wardle@ mit.edu 


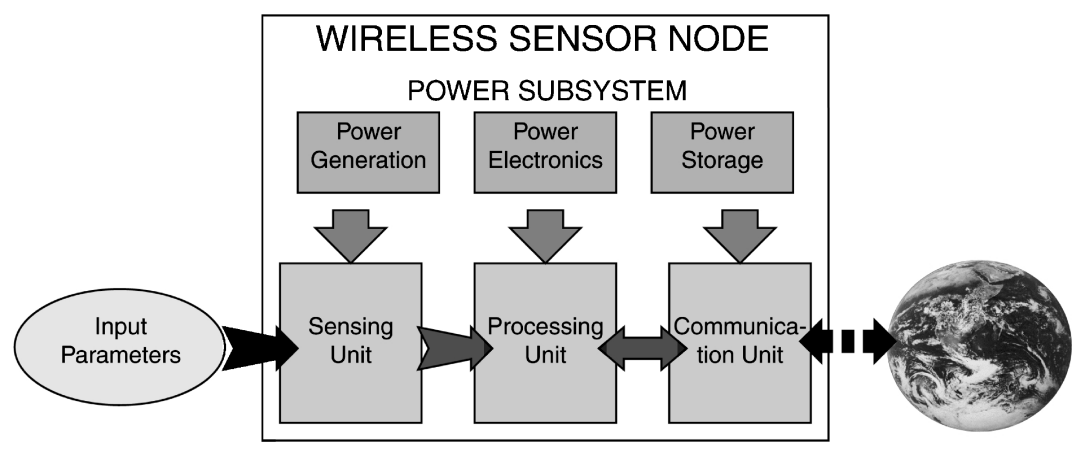

Figure 1. Wireless sensor node architecture.

problems [4]. A node is defined as "a single physical device consisting of a sensor, a transceiver, and supporting electronics, and which is connected to a larger wireless network" [5]. The basic architecture of a node is illustrated in Fig. 1. Applications envisioned for these node-networks include building environment control, warehouse inventory and supply chain control, identification and personalization (RFID tags), the smart home [6], structural health monitoring (aerospace and automotive sectors), agricultural automation, and homeland security applications.

Advances in low power DSP's (Digital Signal Processors) and trends in VLSI (Very Large Scale Integration) system-design have reduced power requirements for the individual nodes [7]. Power consumption of tens to hundreds of $\mu \mathrm{W}$ is predicted $[6,8-10]$ and a current milli-scale commercial node has an average power consumption of 6-300 $\mu \mathrm{W}$, depending on the application and/or mode of operation [11]. This lowered power requirement has made selfpowered sensor nodes a possibility. The power supplies envisioned for these nodes will convert ambient energy into usable electric energy, and therefore can be self-sustaining.

The power source selected for a node will be governed by the specific application. General considerations when selecting a power source for a node include: node network lifetime, cost and size of nodes, node placement and resulting ambient energy availability, and communication requirements. Power or energy sources for nodes can be divided into two groups: sources with a fixed energy density (e.g., batteries) and sources with a fixed power density (normally ambient energy harvesters). These source types are compared in Fig. 2. Fixed energy density sources clearly have limited life-the source either needs to be replaced or the fuel replenished from time to time. Many applications are envisioned where maintenance and repair will not be desired or even possible (e.g., where the sensor is embedded for structural monitoring). Thus, it is desirable to extend the power source life to match that of the application. As 


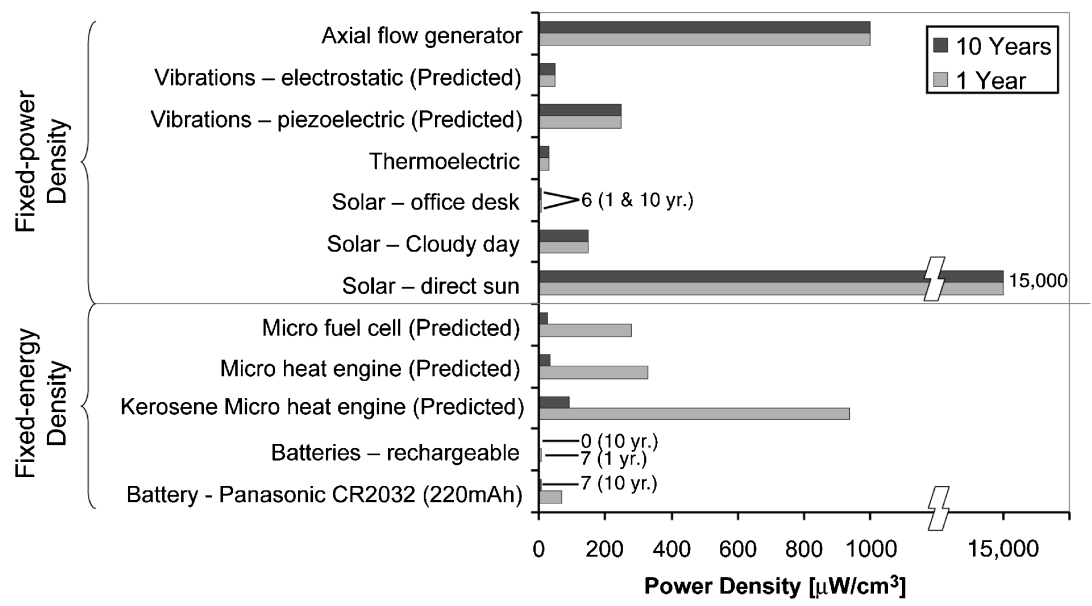

Figure 2. Comparison of fixed-energy and fixed-power density sources for nodes. Adapted from [1, 5, 12, 20, 27-29].

applications such as embedded infrastructure monitoring could span decades, ambient energy harvesters are well suited in these cases.

Many mechanisms of ambient energy scavenging have been investigated, falling into four major categories: solar energy, thermoelectric [12-15], acoustic, and mechanical vibrations. Mechanical vibration energy harvesters can be divided into two groups: non-resonant and resonant devices. The former are applied to very low frequency vibrations [16-18]. Resonant mechanical vibration energy harvesting is the scheme under investigation here and three conversion mechanisms exist: electrostatic [5, 7, 19, 20], electromagnetic [5, 21-26], and piezoelectric.

Some experimental and predicted results on vibration harvesting have been published, and are summarized in Table 1. From the published results it is clear that the power generated varies greatly, according to device size, type, and input vibration parameters. The device sizes vary from the micro-scale $\left(0.01 \mathrm{~cm}^{3}\right)$ to the macro-scale $\left(75 \mathrm{~cm}^{3}\right)$. A normalization scheme can be used to compare the performance and efficiency of the devices relatively. One method is to report the power density $\left(\mathrm{W} / \mathrm{cm}^{3}\right.$ or $\left.\mathrm{W} / \mathrm{kg}\right)$. However, it was found that generally the volume and/or mass are not clearly documented in the literature. Further, the device volume given generally does not specify whether the complete power subsystem is included, or only the power generation unit. The best method for comparing devices would be with an efficiency parameter. From the basic harvester model (see section on ambient vibrations), the power extracted is a function of the input vibration parameters (both amplitude and frequency), the device mass, and the damping ratios (electrical and mechanical). A relative comparison of devices would be possible if all these parameters were made 
Table 1

Previous vibration energy harvester work: simulated and experimental results

\begin{tabular}{|c|c|c|c|c|c|c|}
\hline \multirow[b]{2}{*}{$\begin{array}{l}\text { Power } \\
{[\mu \mathrm{W}]}\end{array}$} & \multirow[b]{2}{*}{$\begin{array}{l}\text { Volume }^{\dagger} \\
{\left[\mathrm{cm}^{3}\right]}\end{array}$} & \multicolumn{3}{|c|}{ Input vibration parameters } & \multirow[b]{2}{*}{$\begin{array}{l}\text { Simulation/ } \\
\text { experiment }\end{array}$} & \multirow[b]{2}{*}{$\begin{array}{l}\text { Type* and } \\
\text { reference }\end{array}$} \\
\hline & & $\begin{array}{c}\text { Frequency } \\
{[\mathrm{Hz}]}\end{array}$ & $\begin{array}{l}\text { Amplitude } \\
{[\mu \mathrm{m}]}\end{array}$ & $\begin{array}{c}\text { Acceleration } \\
{\left[\mathrm{m} / \mathrm{s}^{2}\right]}\end{array}$ & & \\
\hline 58 & 0.5 & 120 & 4.00 & 2.25 & Simulation & ES, [20] \\
\hline 5.6 & N/A & 2520 & N/A & N/A & Simulation & ES, [7] \\
\hline 200 & 75 & 28 & 6.46 & 0.20 & Experiment & EM, [21] \\
\hline 3600 & 75 & 28 & 32.3 & 1.00 & Experiment & EM, [21] \\
\hline 1 & 0.025 & 70 & 30.0 & 5.80 & Simulation & EM, [22] \\
\hline 100 & 0.025 & 330 & 30.0 & 12.0 & Simulation & EM, [22] \\
\hline 530 & 0.24 & 322 & 25.0 & 102 & Experiment & EM, [23] \\
\hline 830 & 1 & 110 & 150 & 71.7 & Experiment & EM, [25] \\
\hline 1000 & N/A & 102 & 8 & 3.29 & Experiment & EM, [24] \\
\hline 186 & 0.5 & 120 & 4.4 & 2.5 & Simulation & $\mathrm{P},[5]$ \\
\hline 260 & 0.5 & 120 & 4.4 & 2.5 & Simulation & $\mathrm{P},[5]$ \\
\hline 242 & 0.5 & 120 & 4.4 & 2.5 & Simulation & $\mathrm{P},[5]$ \\
\hline 260 & 0.5 & 120 & 4.4 & 2.5 & Simulation & $\mathrm{P},[5]$ \\
\hline 60 & N/A & 100 & 5.7 & 2.25 & Experiment & $\mathrm{P},[5]$ \\
\hline 2 & 0.9 & 80 & N/A & N/A & Experiment & $\mathrm{P},[42]$ \\
\hline 1 & 0.01 & 13,900 & 0.014 & 107 & Experiment & $\mathrm{P},[51]$ \\
\hline 900 & 2 & 30 & N/A & N/A & Experiment & $\mathrm{P},[36]$ \\
\hline
\end{tabular}

${ }^{\dagger}$ Device size does not include power electronics. This parameter is oftentimes not documented, but in some cases an estimate of the volume can be made.

* $\mathrm{ES}$ is electrostatic, $\mathrm{EM}$ is electromagnetic, and $\mathrm{P}$ is piezoelectric.

available. Perhaps most importantly, input vibration magnitude and frequency must be documented as the power output (converted power) obviously depends on the power input.

Interest in the application of piezoelectric energy harvesters for converting mechanical energy into electrical energy increased dramatically in recent years, though the idea is not new. An overview of research in this field has recently been given by Sodana et al. [30]. In early work, the predicted power output of a poly-vinylidene fluoride (PVDF) unimorph was so small that it was not a feasible power source at the time [31]. The application of piezoelectric elements to vibration damping (both active and passive) has received much attention [32]. Some authors proposed using the energy extracted from the system to power sensors or electronics [33-35], instead of dissipating the energy through resistive heaters or other dissipative elements. When an energy harvester is applied to a system, structural damping can be achieved [36-38]. 
With the decrease in power requirements for sensor nodes, the application of piezoelectricity to energy harvesting has become viable. Piezoelectric elements in several geometries have been applied for this purpose. The most common is the cantilever beam configuration [5, 24, 26, 36, 37, 39-43] and is the focus of this work. A power density for this type of harvester was predicted to be the highest of the three conversion mechanisms, as high is $250 \mu \mathrm{W} / \mathrm{cm}^{3}$, for a micro-scale device [5], including the complete power subsystem. Other harvesting schemes using piezoelectric elements include membrane structures to harvest energy from pulsing pressure sources [44-46] and converting energy from walking [47, 48]. Research focusing on the power electronics to optimize the transfer of energy from the piezoelectric element to the storage device has also been undertaken $[7,9,49,50]$.

\section{MEMS PIEZOELECTRIC VIBRATION ENERGY HARVESTER (MPVEH)}

This research focuses on a micro-scale vibration energy harvester, applicable to powering a micro-scale sensor node. A cantilever beam configuration was chosen for its simplicity, compatibility with MEMS manufacturing processes, and its low structural stiffness. The beam configuration is a unimorph structure consisting of a structural layer, a single piezoelectric element/layer, and a top interdigitated electrode, as illustrated in Fig. 3. The asymmetric layered design is a consequence of the current MEMS manufacturing process used, as described in the preliminary design section. The process was chosen since a low cost device, integrable with the MEMS fabrication of other subsystems, is desired. However, using a MEMS fabrication process for manufacture imposes definite limits on the size of the device: as size scales down, the resonance frequency of the device scales up. A low resonant frequency is desired since ambient vibration sources (see section on ambient vibration sources) have significant vibration components in the frequency range below $300 \mathrm{~Hz}$. However, designing a MEMS device with the resonant frequency below $100 \mathrm{~Hz}$ can be problematic [5]. For these reasons, a target frequency range of 100-300 Hz was chosen. Available MEMS manufacturing limits the beam length to around $1 \mathrm{~mm}$, such that a proof mass is needed to reduce the natural frequency of the device.

For an initial analysis, only static device failure will be considered. Mechanical fatigue in MEMS devices is oftentimes not an issue given the materials used in micro-fabrication, [52, 53]. However, fatigue (especially in the piezoelectric element) will be considered in the future. Finally, the nominal voltage output from the device has been set to approximately $3 \mathrm{~V}$. This is an electronics industry standard which was likely a consequence of the requirements set by the rectifier circuit [24] and/or to minimize switching losses [24, 50]. Spacing of the interdigitated electrodes allows the output voltage to be controlled. 

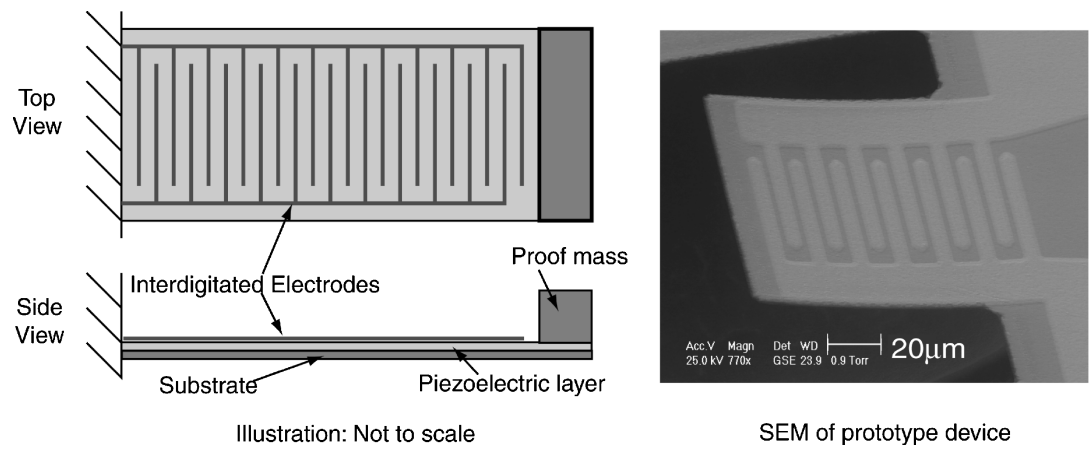

Figure 3. Illustration of MPVEH unimorph configuration (left) and SEM of a prototype device (right).

\section{AMBIENT VIBRATION SOURCES AND MEMS HARVESTERS: INTERPRETATION SCHEME, OPERATING POINT, AND SUMMARY}

In order to better understand the characteristics of low-level ambient vibrations, vibrations measurements for a variety of everyday objects were taken. The purpose of the measurements was to get a quantitative indication of the frequency range and magnitude of vibrations from these sources. 14 conditions of eight separate sources were analyzed in total, for the frequency range of 10$1,000 \mathrm{~Hz}$. Interpretation schemes, utilizing a simple but effective 1-D dynamic model for optimal harvesting point selection, were developed and it was found that macro- and micro-systems require separate schemes since the dominating damping mechanisms for these systems vary. This is in contrast to previous findings in the literature where the mechanisms of the damping were ignored and/or assumed independent of frequency. For micro devices, the operating environment will further influence the selection of a vibration peak to target. Lastly, ambient vibration sources generally exhibit multiple peaks of significant power, often at much higher frequencies. This observation motivates an investigation of the effect of other device resonance modes (e.g., higher beam modes) on the power generation of the system in the sections to follow.

\section{Interpretation Scheme}

A basic 1-D model, as illustrated in Fig. 4, is used to analyze the power generated from a vibration energy harvester to understand conversion, as proposed by $[22,54]$. This model is strictly valid only for harvesters where the electrical damping term is linear and proportional to the velocity (e.g., certain electromagnetic converters [5]), but is useful in understanding the relative importance 


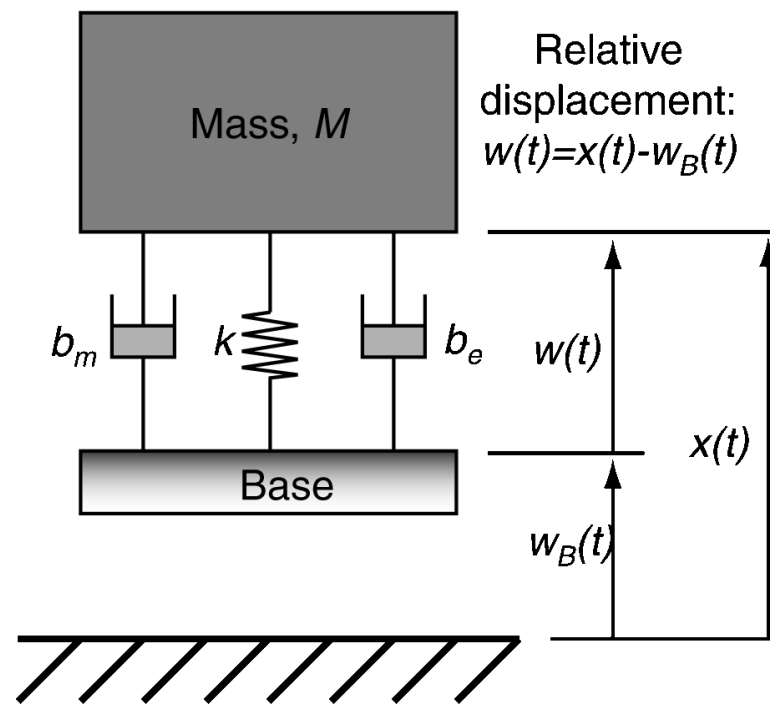

Figure 4. Illustration of basic 1-D model of vibration harvester.

of system (structural and electrical) and input parameters on power extracted. The electrical energy is extracted from the mechanical system, which is excited by a mechanical input. This extraction is not necessarily linear, or proportional to velocity, however, it is a dissipative process and can generally be viewed as electrical damping.

The dynamics of the system in Fig. 4 are described through Eq. (1).

$$
\ddot{w}+2\left(\zeta_{m}+\zeta_{e}\right) \omega_{N} \dot{w}+\omega_{N}^{2} w=-\ddot{w}_{B}
$$

The natural frequency is defined as $\omega_{N}=\sqrt{k / M}$, and the damping ratio is related to the damping coefficient, $b$, through $b=2 M \omega_{N} \zeta$. The total damping, $\zeta_{T}$, is the sum of the mechanical and electrical damping ratios, $\zeta_{m}$ and $\zeta_{e}$ respectively. The base excitation is $w_{B}(t)=W_{B} e^{i \omega t}$, where $W_{B}$ is the magnitude of the base displacement, and $\omega$ the base input frequency. The electrical power extracted through the damping element can be determined, $\left|P_{e}\right|=\frac{1}{2} b_{e} \dot{w}^{2}$, and can be written in terms of the input vibration parameters. $\ddot{W}_{B}$ is the magnitude of the base acceleration and is related to the base displacement magnitude through $\ddot{W}_{B}=\omega^{2} W_{B}$ for harmonic inputs, as is assumed here. Use was also made of the definition of the frequency ratio, $\Omega=\omega / \omega_{N}$.

$$
\left|P_{e}\right|=\frac{M \zeta_{e} \Omega^{2} \omega^{4} W_{B}^{2}}{\omega_{N}\left[\left(1-\Omega^{2}\right)^{2}+\left(2 \zeta_{T} \Omega\right)^{2}\right]}=\frac{M \zeta_{e} \Omega^{2} \ddot{W}_{B}^{2}}{\omega_{N}\left[\left(1-\Omega^{2}\right)^{2}+\left(2 \zeta_{T} \Omega\right)^{2}\right]}
$$


The power generated can be maximized by determining the optimal operating frequency ratio.

$$
\left(\omega_{o p t, P e} / \omega_{N}\right)^{2}=2\left(1-2 \zeta_{T}^{2}\right) \pm \sqrt{4\left(2 \zeta_{T}^{2}-1\right)^{2}-3}
$$

When the total damping ratio is small, Eq. (3) suggests $\omega_{\text {opt }} \approx \omega_{N}$. A second optimum around $\omega_{\text {opt }} \approx \sqrt{3} \omega_{N}$ is suggested by Eq. (3), but is a local minimum. Since the total damping will typically be small, it is sufficient to let $\omega_{\text {opt }}=\omega_{N}$, or $\Omega=1$.

Next, the power generated can be maximized with respect to the electrical damping ratio. The optimum is calculated as:

$$
\zeta_{e, o p t}=\sqrt{\zeta_{m}^{2}+\left(\Omega^{2}-1\right)^{2} / 4 \Omega^{2}}
$$

When $\Omega=1$, the optimal electrical damping of $\zeta_{e, o p t}=\zeta_{m}$ is obtained. These simplifications can be substituted into Eq. (2) to obtain:

$$
\left|P_{e}\right|_{\max }=\frac{M \ddot{W}_{B}^{2}}{16 \zeta_{e, o p t} \omega_{N}}
$$

In order to interpret this result, the damping ratio needs to be investigated more closely since $\zeta_{e, o p t}=\zeta_{m}$ for optimal power generation. This damping ratio has four dominant components for a MEMS-scale cantilever beam structure [26, 55-57]: drag force (airflow force), squeeze-force, support losses, and structural damping. These four components can be modeled by adding linearly, as in Eq. (6) and are defined in Eqs. (7-10).

$$
\begin{aligned}
\zeta_{m} & =\zeta_{m, \text { drag }}+\zeta_{m, \text { squeeze }}+\zeta_{m, \text { struct }}+\zeta_{m, \text { sup }} \\
\zeta_{m, \text { drag }} & =\frac{3 \pi \mu d+\frac{3}{4} \pi b^{2} \sqrt{2 \rho_{\text {air }} \mu \omega}}{2 \rho_{\text {beam }} b h l \omega} \\
\zeta_{m, \text { squeeze }} & =\frac{\mu b^{2}}{2 \rho_{\text {beam }} g_{0}^{3} h \omega} \\
\zeta_{m, \text { sup }} & =0.23 h^{3} / l^{3} \\
\zeta_{m, \text { struct }} & =\eta / 2
\end{aligned}
$$

Here $\mu$ is the viscosity of air, $\rho_{\text {air }}$ and $\rho_{\text {beam }}$ are the densities of air and the beam structure respectively, $\eta$ is the structural damping factor for the beam material, $g_{0}$ is the gap between the bottom surface of the beam and a fixed floor, $h$ is the height of the beam, $b$ is the beam width, and $l$ is the length. The operating frequency, $\omega$, coincides with the natural frequency. 
For a micro-scale device, the drag force damping term is dominant when the device is operated in free space (e.g., not close to a wall) and under atmospheric conditions. When the device is operated near a wall, the squeeze-force damping term becomes dominant. When the device is operated in free space in a vacuum, the structural damping term becomes dominant. The structural damping factor is determined empirically, so for the purpose of the analysis that follows, $\eta=5.0$ $10^{-6}$ was used (obtained from [56]).

For the proposed micro-scale design (a cantilever beam operating in free space under atmospheric conditions), the only significant source of damping is due to the drag force. This component has two terms, $\zeta_{m, \text { drag }} \propto \alpha / \omega_{N}+$ $\beta / \sqrt{\omega_{N}}$, where $\alpha$ and $\beta$ are constants. Thus, for the purposes of the analysis, the damping-frequency relation is $\zeta_{e, o p t}=\zeta_{m} \propto 1 / \omega_{N}$. This relation also holds for the squeeze-force damping term. Substituting this result into Eq. (5) it is concluded that the input vibration parameter that most influences the generated power for the current conditions is the acceleration (see Eq. (11)). Thus, when comparing different sources of vibration for a micro-scale device under atmospheric conditions, it is important to maximize the acceleration.

$$
\left|P_{e}\right|_{\max } \propto M \ddot{W}_{B}^{2}
$$

When the micro-scale device is operated in vacuum, the dominant damping components are independent of frequency. The vibration input will be related to the power generated through:

$$
\left|P_{e}\right|_{\max } \propto \frac{M \ddot{W}_{B}^{2}}{\omega_{N}}
$$

In summary, the optimal operating point for power harvesting is a function of the harvesting device/system and is strongly influenced by the dominant damping mechanism in the system. The damping mechanism for a micro-scale device is influenced by whether the device is in vacuum or atmospheric conditions for the current configuration. As devices scale down, the surface-fluid interactions become dominant over inertial effects for microscopic devices. These fluidicdamping mechanisms are generally dependent on frequency, which must be accounted for when analyzing the generated power. On the other hand, surfacefluid interactions are negligible for macroscopic systems, and the dominant damping mechanisms (structural damping and support losses) are generally independent of frequency [57].

\section{Operating Point Selection}

When selecting the vibration peak (in terms of acceleration and frequency) to design a MPVEH for maximum power generation, the maximum value of the input acceleration squared $\left(\ddot{W}_{B}^{2}\right)$ must be considered as the device is to be 
operated in atmospheric conditions. Equivalently, the input parameters can be written in terms of the input frequency and the displacement, $\omega^{4} \ddot{W}_{B}^{2}$. To facilitate this selection, lines of constant "reference power" can be added to any measured acceleration-frequency plots. Reference power is defined as $\left|P_{e}\right|_{\max }$ in Eq. (5), at the acceleration and frequency of the highest acceleration peak above $100 \mathrm{~Hz}$ (see Fig. 5). These constant power lines indicate the maximum contribution to the power generated from the input spectrum, assuming damping ratio-optimized resonant harvesters of equal mass. When the MEMS-scale device is operated in vacuum, the damping ratio is independent of the frequency, and the ratio of acceleration squared to the frequency should be used to interpret the measurement data (Fig. 5). In some cases, the optimal operating peak can have a lower acceleration than other peaks.

Two other schemes for the interpretation of the measurement data have been put forward. In the first, the peak power generated is written as in Eq. (2) (with $\Omega=1$ ), but the frequency dependence of the damping term is not considered $[5,20]$. The second writes the power generated both in terms of input and output parameters, which can be simplified to obtain Eq. (2) (with $\Omega=1$ ), and similarly does not account for the damping-frequency dependence for a MEMS device [58].

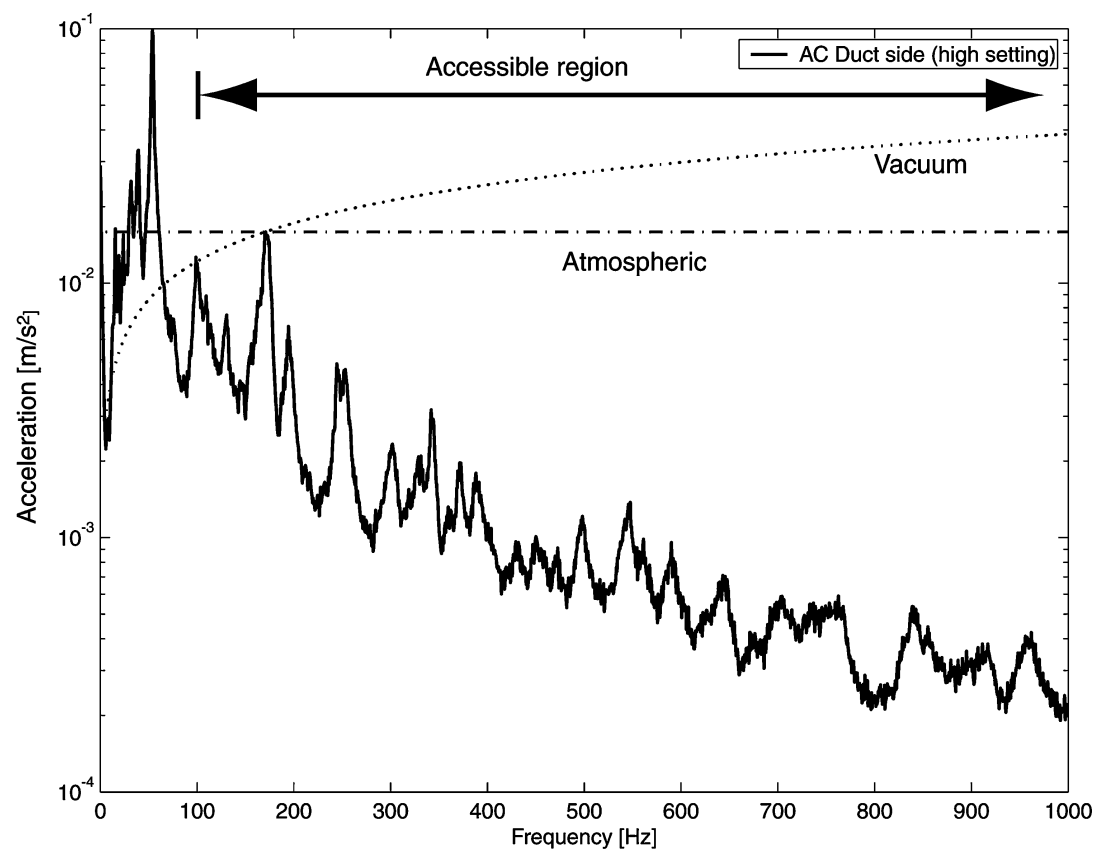

Figure 5. Interpreting ambient acceleration-frequency plots to determine target acceleration peak for the MPVEH under atmospheric conditions: A/C duct side example. 
Another important consideration is the lower limit in natural frequency obtainable with a MEMS device. Consistent with our conclusions, others (e.g., [5]) have found it difficult to design a MEMS device with a resonant frequency below $100 \mathrm{~Hz}$ (as size scales down, resonant frequency scales up). The lower limit for viable vibration peaks has been set to $100 \mathrm{~Hz}$ for the current investigation, thus defining the "accessible region" to be above $100 \mathrm{~Hz}$ (see Fig. 5).

\section{Summary of Low-Level Ambient Sources}

The interpretation scheme for a MPVEH device operated in atmospheric conditions, $\left|P_{e}\right|_{\max } \propto \ddot{W}_{B}^{2}$, as discussed in the previous section, is used to identify three acceleration peaks for each source that are listed in Table 2 . The first peak has the maximum power content (e.g., the highest acceleration squared) and is referred to as the 'Highest Power Peak,' or HPP. The 'Reference Peak,' RP, is the highest power peak in the accessible region (i.e., above $100 \mathrm{~Hz}$ ). The 'Alternate Peak,' AP, is a secondary peak in the accessible region. For some of the sources, the HPP and the RP are the same.

From Table 2 it can be seen that the ranges and levels of ambient vibrations differ greatly: for HPP, the levels varied from $10^{-3} \mathrm{~m} / \mathrm{s}^{2}$ to around $4 \mathrm{~m} / \mathrm{s}^{2}$. However, not all these peaks are accessible (e.g., above $100 \mathrm{~Hz}$ ), and RP values range from $10^{-4} \mathrm{~m} / \mathrm{s}^{2}$ to $4 \mathrm{~m} / \mathrm{s}^{2}$.

These results show good agreement with published ambient vibration data, e.g. [5]. Upon comparing RP and AP values, two important observations can be made. Firstly, in 7 of the 14 cases investigated, an AP was identified at a lower frequency than the RP. The significance of this becomes clear when the device is operated in vacuum. As is illustrated in Fig. 5, the constant power lines for a device operated in vacuum drop to zero as the frequency decreases. This is because the power generated is inversely proportional to the vibration frequency. From the example it is clear that the AP will have the same power content as the RP. If the operating environment is vacuum, the optimal harvesting point for a MEMS-scale beam harvester will not correspond to the highest vibration level peak (RP as defined here), but will be at a lower level and frequency. A reference vibration of $\ddot{W}_{B}=4.2 \mathrm{~m} / \mathrm{s}^{2}$ at $150 \mathrm{~Hz}$ is used for the preliminary design of a low-level MPVEH device in a later section (vibrations measured on a microwave oven side panel).

The second observation is that some sources exhibit peaks with comparable power content at much higher frequencies. See for example source 12 (car hood at $3000 \mathrm{rpm}$ ) in Table 2. The higher frequency peak can excite a second or third resonance mode of the structure and strain cancellation (and therefore power loss) in the harvester device is possible. The example reference peak has $0.257 \mathrm{~m} / \mathrm{s}^{2}$ acceleration at $148 \mathrm{~Hz}$, and an alternate peak at $881 \mathrm{~Hz}$ with an acceleration of $0.102 \mathrm{~m} / \mathrm{s}^{2}$. This finding prompted an investigation into the effect of higher frequency modes of the beam structure when aligned to an alternate peak of the source vibration. 


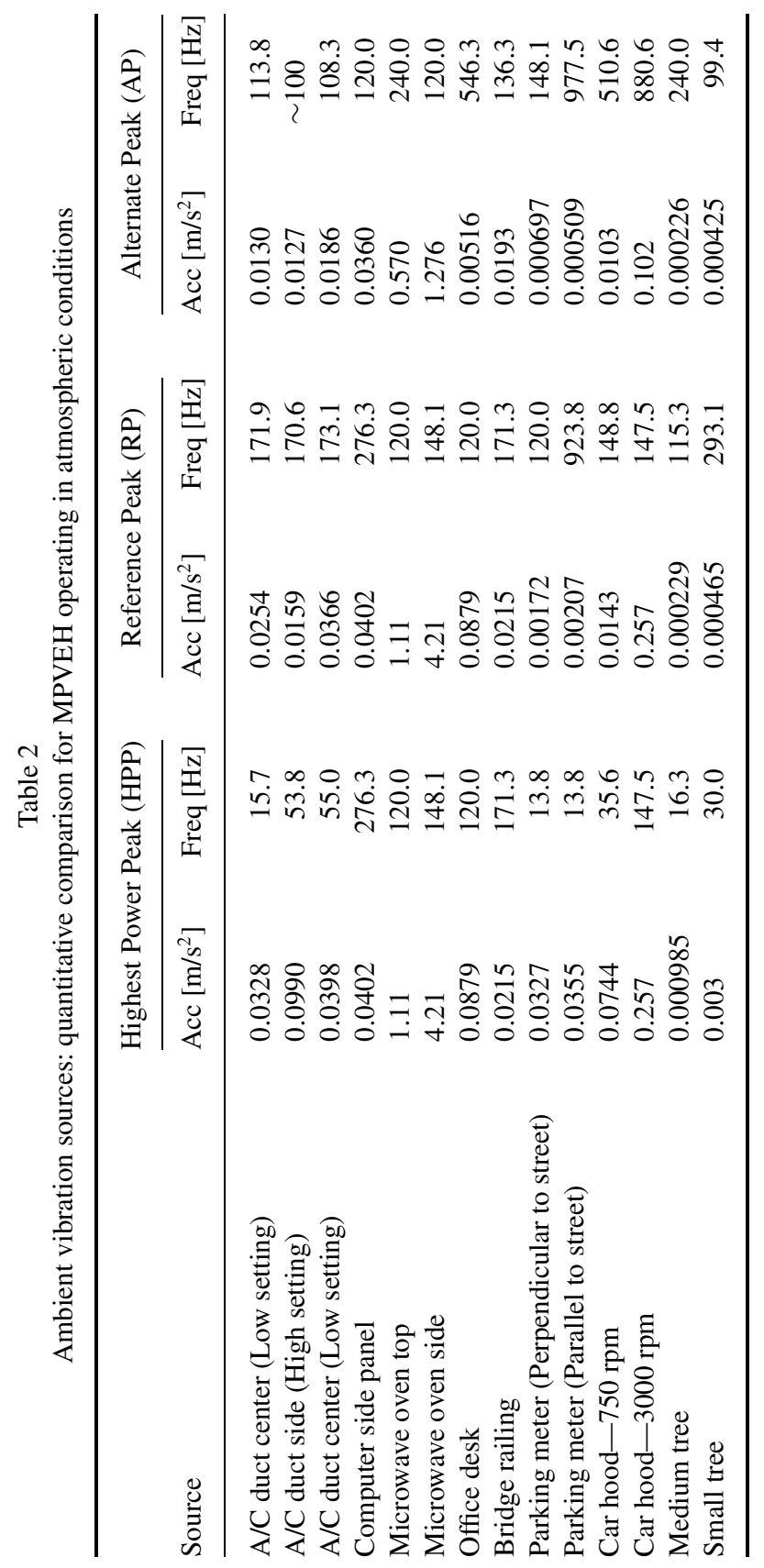


As an example, a simple cantilever beam configuration was created with the first resonance frequency at $140 \mathrm{~Hz}$ and the second resonance frequency at $875 \mathrm{~Hz}$. Thus, with the variability of vibration sources, it is possible for the source alternate peak and the second resonance peak of the beam to align. To investigate strain cancellation for this simple cantilever, a modal analysis of the device with two input vibration components was conducted. The first component was aligned to the first resonance of the device (as per design), and the second component coincided with the second resonance of the beam. The vibration level of the second frequency component was varied to analyze the effect on the developed strain. Please refer to Fig. 6 for maximum axial strain vs. the beam length at maximum tip displacement under these assumptions. The power of the second or alternate input peak is zero, equal to, and half the power of the reference peak, respectively. The strain developed over the first region of the beam (near the base) increases with the additional excitation, whereas the strain is decreased towards the tip. Assuming that the power scales linearly with the strain, it is necessary to look at the total area under the strain

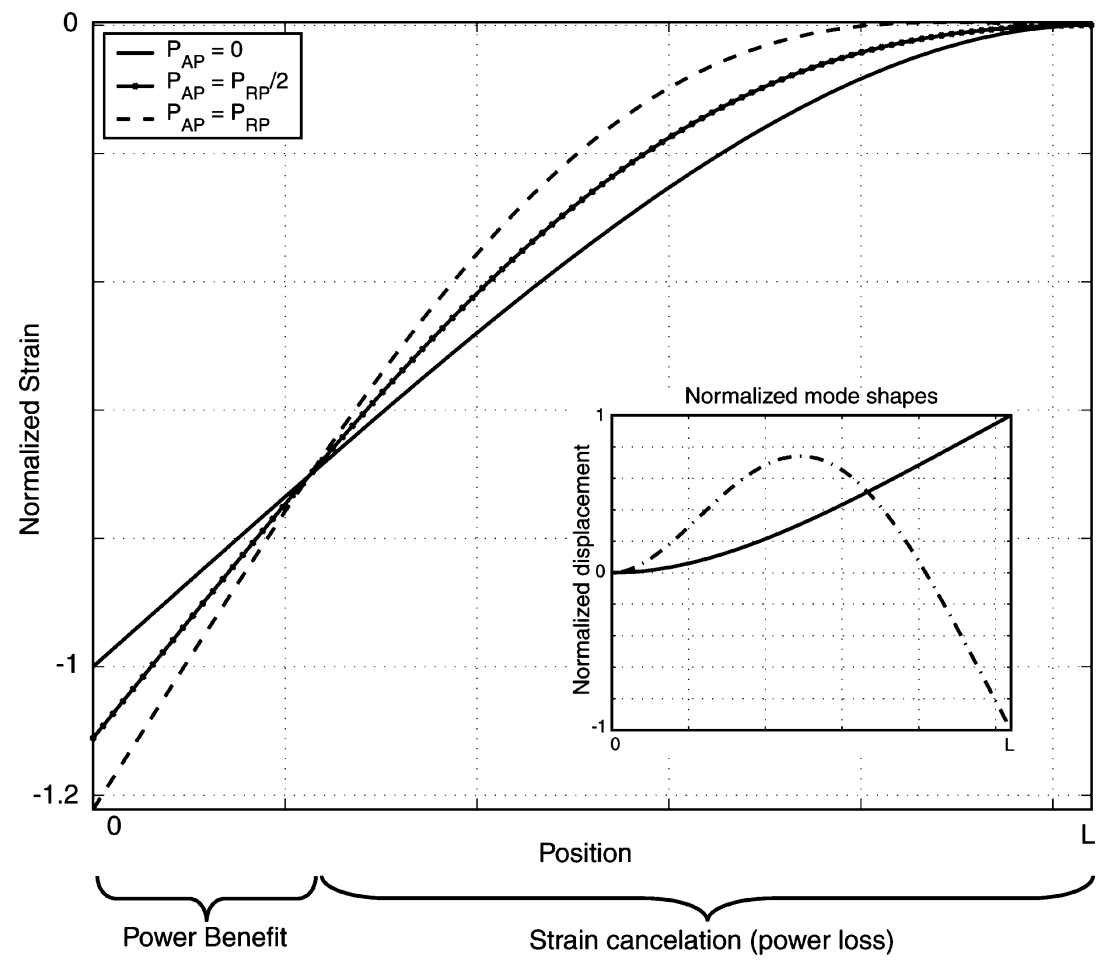

Figure 6. Strain distribution for a beam excited by multiple input vibration components, illustrating net power loss. 
curve. Since the second mode causes the total area to decrease, the total power will decrease too, assuming that the electrodes cover the whole surface. Thus, it is possible to identify an optimal electrode length in the region where the strain is increased, but that is not affected by the cancellation of strain, for this simple case. Furthermore, the second mode contributes by increasing the maximum developed strain at the base of the beam, affecting the static failure design of such devices. For the purpose of the analysis, it is assumed that the mode deflections due to the two inputs are in phase. Should this not be the case, a different final strain distribution will be obtained, but the important conclusion that strain cancellation is possible due to the influence of multiple input vibration components remains.

\section{MODELING: 1-D ANALYTICAL MODEL, CANTILEVER BEAM MODEL}

Coupled electromechanical models are developed, validated, and later applied to the preliminary design and performance prediction of a low-level MPVEH. A basic 1-D accelerometer-type closed-form model is shown to characterize beam configuration harvesters adequately by comparison to more rigorous modal analyses. Unimorph and bimorph beam configurations, as well as $\{3-1\}$ and \{3-3\} actuation modes (using interdigitated electrodes) are considered, as well as beams with proof masses at the free end.

\section{Power-Optimized 1-D Electromechanical Analytic Model}

A closed-form coupled electromechanical 1-D model is developed that captures the basic response of piezoelectric vibration harvesters and is useful in interpreting prior and more detailed 2-D beam models presented later. The model is illustrated in Fig. 7 and consists of a piezoelectric element excited by a base input, $w_{B}$. The piezoelectric element has a mass $m_{p}$ and is connected to a power-harvesting circuit, modeled simply as a resistor. A proof mass, $M$, is also considered. Note that the entire structure is electromechanically coupled in this example, whereas in energy harvesters such as unimorph/bimorph beams, a portion of the structure will be a non-piezoelectric substrate.

Using the 1-D configuration, we can simplify the linear elastic constitutive relations [59, 60], Eq. (13), to Eq. (14):

$$
\begin{aligned}
\left\{\begin{array}{l}
\mathbf{T} \\
\mathbf{D}
\end{array}\right\} & =\left[\begin{array}{cc}
\mathbf{c}^{E} & -\mathbf{e}^{t} \\
\mathbf{e} & \varepsilon^{S}
\end{array}\right]\left\{\begin{array}{l}
\mathbf{S} \\
\mathbf{E}
\end{array}\right\} \\
T_{3} & =c_{33}^{E} S_{3}-e_{33} E_{3} \\
D_{3} & =e_{33} S_{3}+\varepsilon_{33}^{S} E_{3}
\end{aligned}
$$




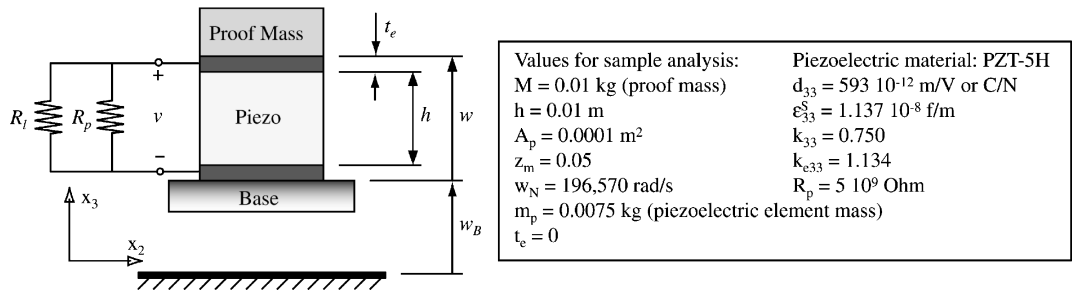

Figure 7. General 1-D model of piezoelectric vibration energy harvester

$\mathbf{D}, \mathbf{E}, \mathbf{S}$, and $\mathbf{T}$ are defined as the electric displacement, electric field, strain, and stress, respectively. $\varepsilon$ is the permittivity of the piezoelectric element and $\mathbf{e}$ is the piezoelectric constant relating charge density and strain. The superscripts $E$ and $S$ indicate a parameter at constant electric field and strain respectively, while superscript $t$ indicates the transpose of the matrix. For the analysis in later sections, superscripts $D$ and $T$ indicate a parameter at constant electric charge and stress, respectively.

From a force equilibrium analysis, the governing equations can be found in terms of the device parameters defined in Fig. 7:

$$
\begin{aligned}
\ddot{w}+2 \zeta_{m} \omega_{N} \dot{w}+\omega_{N}^{2} w-\omega_{N}^{2} d_{33} v & =-\ddot{w}_{B} \\
R_{e q} C_{p} \dot{v}+v+m_{e f f} R_{e q} d_{33} \omega_{N}^{2} \dot{w} & =0
\end{aligned}
$$

$\zeta_{m}$ is the mechanical damping ratio, $\omega_{N}$ is the natural frequency of the device and $m_{\text {eff }}=M+\frac{1}{3} m_{p}$ is the approximate effective mass. The overhead dot indicates the time derivative. The equations are written in terms of d (the piezoelectric constant relating charge density per stress) because this piezoelectric material parameter is more readily available and is related to $\mathbf{e}$ through $\mathbf{d}=\left(\mathbf{c}^{E}\right)^{-1} \mathbf{e}$. The resonance frequency of the 1-D device is given by $\omega_{N}^{2}=c_{33}^{E} A_{p} / m_{e f f} h$, where $A_{p}$ is the area of the electrodes (or the piezoelectric element). The equivalent resistance, $R_{e q}$, is the parallel resistance of the load and the piezoelectric leakage resistance, $R_{l}$ and $R_{p}$ respectively. In general, the leakage resistance is much higher than the load resistance [61], so that $R_{e q} \approx R_{l}$. Lastly, the capacitance is defined in terms of the constrained permittivity, $C_{p}=\varepsilon_{33}^{S} A_{p} / h$.

Using Laplace transforms, the governing equations can be evaluated and the magnitudes of the displacement, voltage, and the power extracted can be determined. These are given in Eqs. (17-19), normalized by the base input acceleration. The dimensionless parameters $\Omega=\omega / \omega_{N}, r=\omega_{N} R_{e q} C_{p}$, and an alternative electromechanical coupling coefficient, $k_{e}^{2}=\frac{k_{33}^{2}}{1-k_{33}^{2}}=\frac{e_{33}^{2}}{c_{33}^{k} \xi_{33}^{S}}$ can be defined [62] to simplify the analysis. $k_{33}$ is the electromechanical coupling 
coefficient relating the output electrical energy to the input mechanical energy.

$$
\begin{aligned}
& \left|\frac{w}{\ddot{w}_{B}}\right|=\frac{1 / \omega_{N}^{2} \sqrt{1+(r \Omega)^{2}}}{\sqrt{\left[1-\left(1+2 \zeta_{m} r\right) \Omega^{2}\right]^{2}+\left[\left(1+k_{e}^{2}\right) r \Omega+2 \zeta_{m} \Omega-r \Omega^{3}\right]^{2}}} \\
& \left|\frac{v}{\ddot{w}_{B}}\right|=\frac{m_{e f f} R_{e q} d_{33} \omega_{N} \Omega}{\sqrt{\left[1-\left(1+2 \zeta_{m} r\right) \Omega^{2}\right]^{2}+\left[\left(1+k_{e}^{2}\right) r \Omega+2 \zeta_{m} \Omega-r \Omega^{3}\right]^{2}}} \\
& \left|\frac{P_{\text {out }}}{\left(\ddot{w}_{B}\right)^{2}}\right|=\frac{m_{e f f} 1 / \omega_{N} r k_{e}^{2} R_{e q} / R_{l} \Omega^{2}}{\left[1-\left(1+2 \zeta_{m} r\right) \Omega^{2}\right]^{2}+\left[\left(1+k_{e}^{2}\right) r \Omega+2 \zeta_{m} \Omega-r \Omega^{3}\right]^{2}}
\end{aligned}
$$

In prior energy harvester work, Eqs. (17-19) have always been simplified by setting $\Omega=1$, which will be shown to miss important aspects of the coupled response (particularly anti-resonance). It is of interest to optimize the power extracted from the source. Again, taking the leakage resistance as much larger than the load resistance, the power can be optimized with respect to the load resistance, $R_{l}$, or equivalently with respect to the dimensionless parameter, $r$.

$$
r_{o p t}=\frac{\Omega^{4}+\left(4 \zeta_{m}^{2}-2\right) \Omega^{2}+1}{\Omega^{6}+\left(4 \zeta_{m}^{2}-2\left[1+k_{e}^{2}\right]\right) \Omega^{4}+\left[1+k_{e}^{2}\right]^{2} \Omega^{2}}
$$

Furthermore, since a piezoelectric element exhibits both open- and shortcircuit stiffness, there will be two optimal power operating frequencies to consider. For the short circuit stiffness we let $R_{l} \rightarrow 0$ in Eq. (19) to obtain:

$$
\Omega_{s c}=1 \quad \text { or } \quad \omega_{S C}=\sqrt{c_{33}^{E} A_{p} / m_{e f f} h}
$$

This has previously been defined as the resonance frequency. For the open circuit analysis we let $R_{l} \rightarrow \infty$ to obtain:

$$
\Omega_{o c}=\sqrt{1+k_{e}^{2}}=\frac{1}{\sqrt{1-k_{33}^{2}}} \quad \text { or } \quad \omega_{O C}=\sqrt{c_{33}^{D} A_{p} / m_{e f f} h}
$$

This frequency is known as the anti-resonance frequency and is governed by the electromechanical coupling coefficient of the device, as expected. Note that $\Omega_{o c}>\Omega_{s c}$. Using these frequency ratios, it is possible to obtain optimal resistances with Eq. (20) at the respective operating points. Power is plotted against the frequency ratio for the values given in Fig. 7 with a base input acceleration of $9.81 \mathrm{~m} / \mathrm{s}^{2}$, or $1 \mathrm{~g}$, in Fig. 8. The thick, solid line forms the envelope of maximum power because the resistance is maximized at all frequency ratios. Switching between the two peaks, corresponding to the resonance and antiresonance frequencies, is achieved by varying the electrical load. The power 


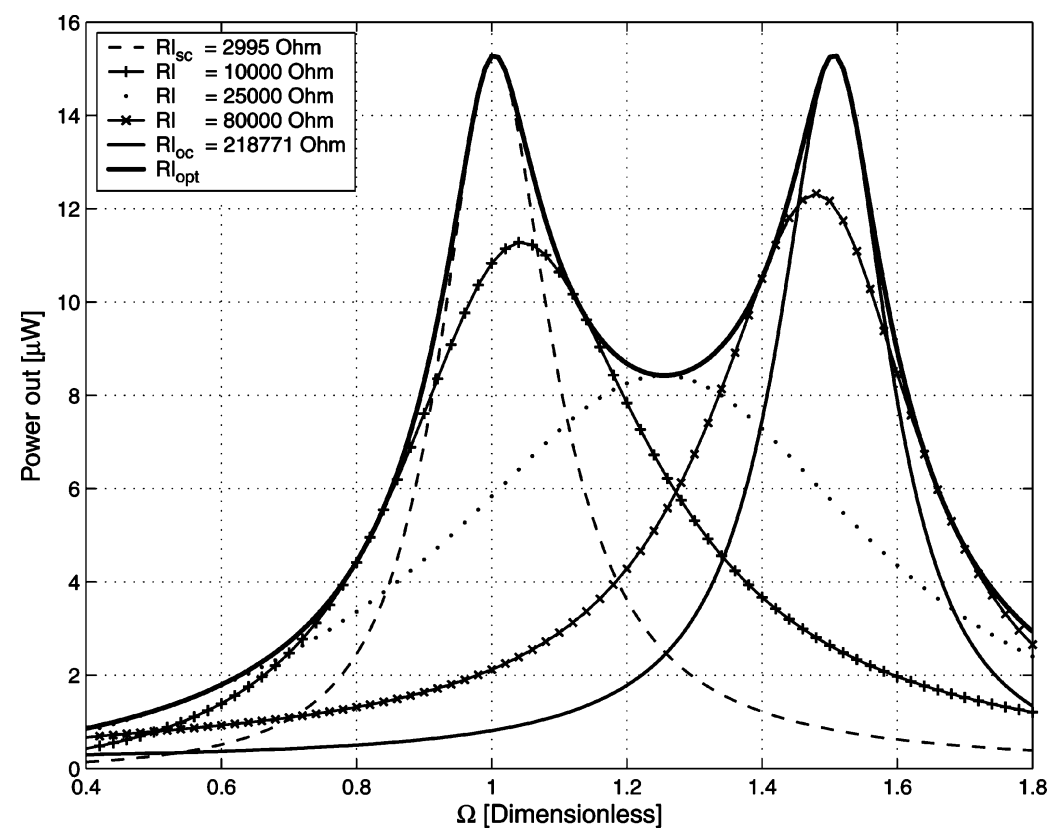

Figure 8. Power vs. normalized frequency with varying electrical load resistance for 1-D model in Fig. 7.

increases as the resonance frequency is approached, and reaches a maximum, before decreasing to a local minimum, which corresponds to a minimum proof mass displacement. The power then increases to a second maximum, corresponding to the anti-resonance frequency. While the power predicted at these peaks is equal, the voltage and current differ significantly.

Next, the relative displacements of the proof mass at the two peaks are compared, as illustrated in Fig. 9. The electrical resistance is still optimized for maximum power, as in Fig. 8. Unlike the power, the displacement is higher at the resonance than at the anti-resonance. Also note that the relative displacement of the proof mass is not minimized at either the resonance or the anti-resonance peak (where the power extracted is maximized), but at an intermediate peak. Operating at the second peak could be advantageous since the proof mass displacement will be smaller, allowing for a smaller device.

The voltage is plotted against the frequency ratio in Fig. 10. As with the displacement, the voltage generated at the two peaks differ, but in the case of the voltage the difference is around an order of magnitude. Since the power generated at these peaks are the same, and power is related to voltage and current, $i$, through $P_{\text {out }}=v i$ (for a resistive load), the current at the first peak will be an order of magnitude higher than at the second peak. The capability of a piezoelectric element to charge a secondary battery was previously investigated 


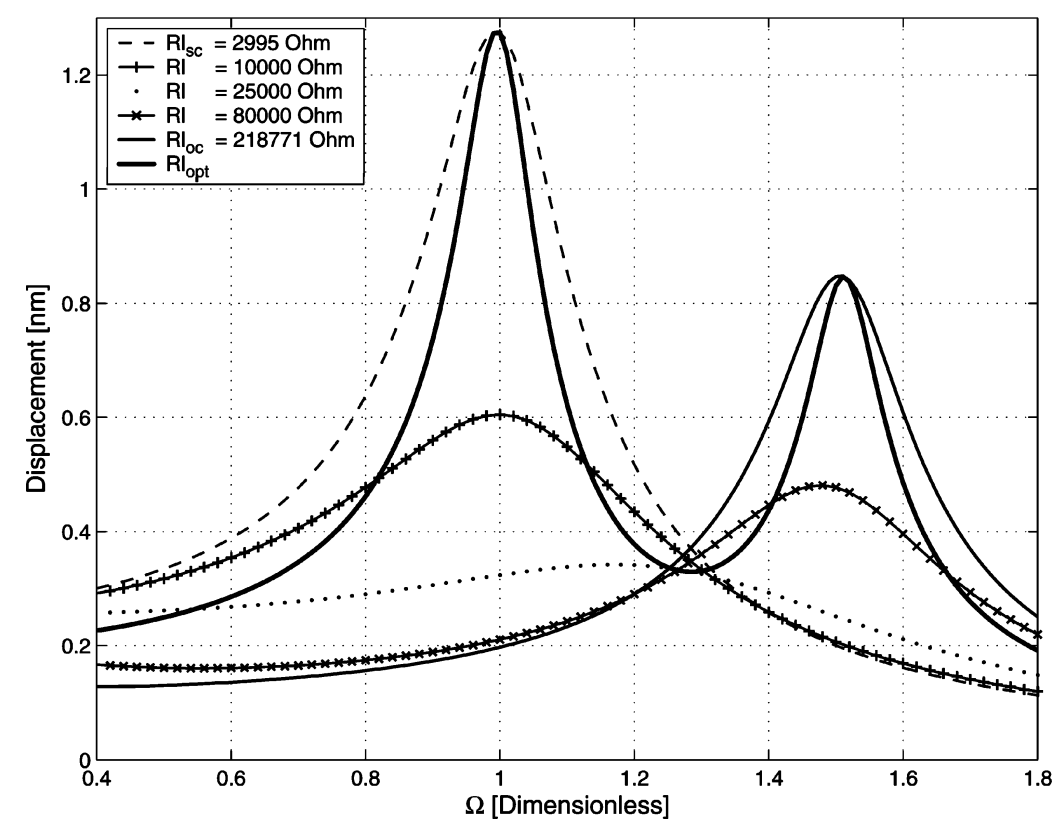

Figure 9. Displacement vs. normalized frequency with varying electrical load resistance for 1-D model in Fig. 7.

[63]. From the study it was concluded that certain piezoelectric elements are not well suited for battery charging applications, as generated current levels are too low. Operation at the resonance frequency could possibly alleviate the problem. Furthermore, the rectifier circuit has a minimum voltage requirement for operation, and the voltage requirement is also governed by the onset of losses at lower voltages [24]. Operating at the anti-resonance frequency could be advantageous in this case.

The frequency ratio is plotted against electrical load resistance in Fig. 11 to clearly show the shift from the resonance to anti-resonance frequencies is clearly observable. The magnitude of $\Omega_{o c}$ vs. $\Omega_{s c}$ is governed by the piezoelectric constant, $\mathbf{d}$, and the stiffness contribution of the piezoelectric element to the device. In the 1-D model, the shift is most pronounced since the piezoelectric element constitutes the entire structure (and thus the stiffness).

To summarize, as the electrical load is increased, the piezoelectric element operating condition is changed from short-circuit to open-circuit. Since the piezoelectric element constitutes the entire structure, there is a significant shift in the frequency as operation is switched between the short- and open circuit conditions. For beams at the macro-scale, this effect is not as pronounced as the piezoelectric element does not contribute significantly to the overall structural stiffness. It is much more important at the micro-scale. The power generated at short- and open-circuit conditions are the equal, but the voltage and current 


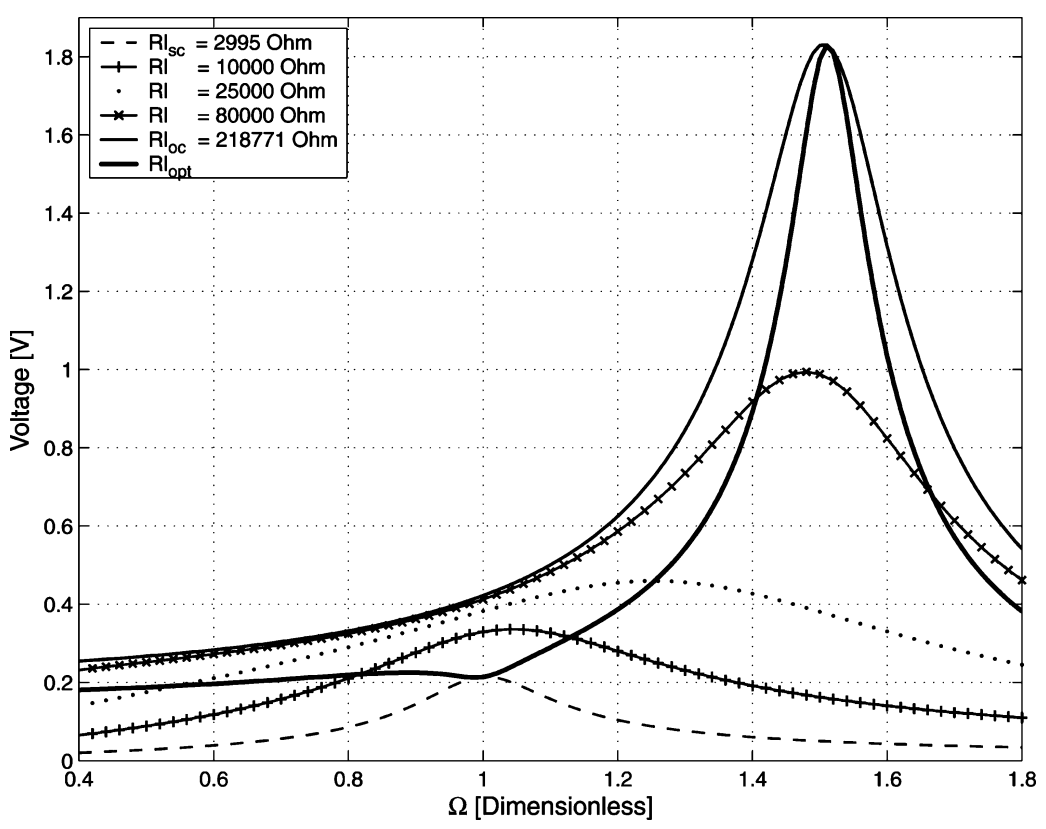

Figure 10. Voltage vs. normalized frequency with varying electrical load resistance for 1-D model in Fig. 7.

developed at the different operating points differ substantially. The displacement of the proof mass of the device is also different at these operating points, as the system will have more damping at the open-circuit condition (due to more electrical damping).

\section{Modeling Unimorph/Bimorph Cantilever Beam Configurations}

As stated earlier, the unimorph cantilever beam configuration was chosen for its geometric compatibility with the MEMS fabrication processes. It is also a relatively compliant structure, allowing for large strains and thus more power generation. The basic configuration of a bimorph is illustrated in Fig. 12 and has the following components: the beam structure, piezoelectric elements, electrodes, and a proof mass if necessary.

In the section to follow, a modal analysis for a base-excited cantilever beam with a mass at the end and a $\{3-1\}$ actuation model of a typical bimorph are developed. The model is then extended to a unimorph configuration operating in the $\{3-3\}$ actuation mode. The model predictions are compared to published experimental and modeling results, and also to experimental work from a prototype MEMS device. Since the coordinate systems for the $\{3-1\}$ and $\{3-3\}$ models are different, two general position variables are introduced: $x_{a}$ indicates 


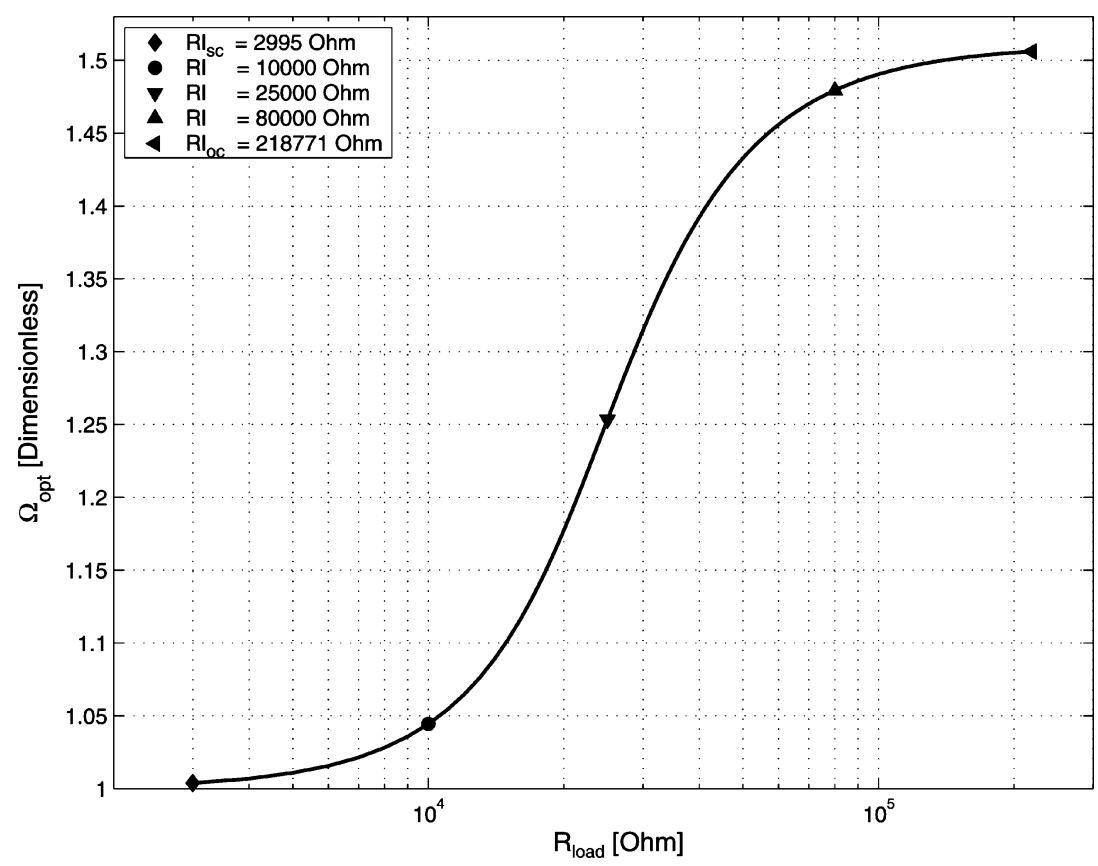

Figure 11. Power-optimal normalized frequency vs. electrical load resistance for 1-D model in Fig. 7.

the axial position (along the length of the beam/structure), and $x_{t}$ indicates the position through the thickness of the beam/structure.

\section{Modeling of a Cantilever Beam with Piezoelectric Elements}

The model for a cantilever beam with piezoelectric elements can be obtained with an energy method approach. An alternative method, which will not be

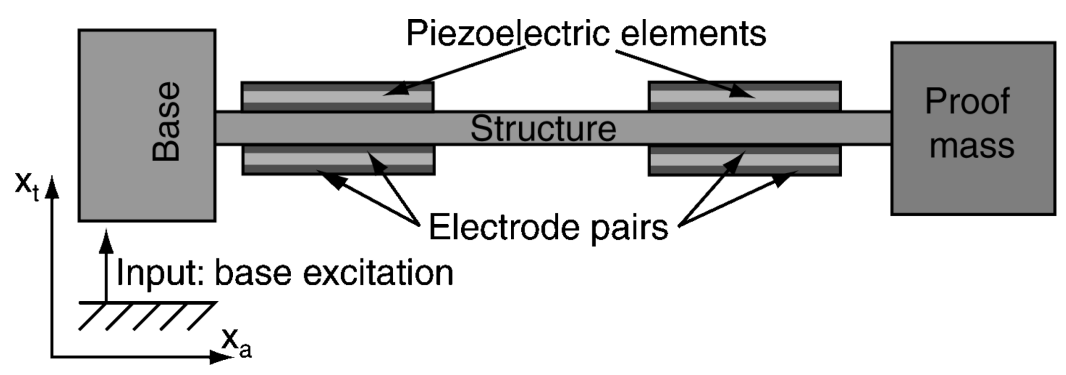

Figure 12. Cantilever bimorph configuration. 
discussed in this paper, is a force equilibrium analysis [64]. The analysis to follow was adapted from [65]. The generalized form of Hamilton's Principle for an electromechanical system, neglecting the magnetic terms and defining the kinetic $\left(T_{k}\right)$, internal potential $(U)$, and electrical $\left(W_{e}\right)$ energies, as well as the external work $(W)$, is given by:

$$
\int_{t 1}^{t 2}\left[\delta\left(T_{k}-U+W_{e}\right)+\delta W\right] d t=0
$$

The individual energy terms are defined as:

$$
\begin{aligned}
T_{k} & =\int_{V s} \frac{1}{2} \rho_{s} \dot{\mathbf{u}}^{t} \dot{\mathbf{u}} d V_{s}+\int_{V p} \frac{1}{2} \rho_{p} \dot{\mathbf{u}} \dot{\mathbf{u}} d V_{p} \\
U & =\int_{V s} \frac{1}{2} \mathbf{S}^{t} \mathbf{T} d V_{s}+\int_{V p} \frac{1}{2} \mathbf{S}^{t} \mathbf{T} d V_{p} \\
W_{e} & =\int_{V p} \frac{1}{2} \mathbf{E}^{t} \mathbf{D} d V_{p}
\end{aligned}
$$

The subscripts $p$ and $s$ indicate the piezoelectric element and the inactive (structural) sections of the beam volume, respectively. The mechanical displacement is denoted by $\mathbf{u}(\mathbf{x}, t)$ and $\rho$ is the density. The contributions to $\mathrm{W}_{e}$ due to fringing fields in the structure and free space are neglected. Considering $n f$ discretely applied external point forces, $\mathbf{f}_{k}(t)$, at positions $\mathbf{x}_{k}$, and $n q$ charges, $q_{j}$, applied at discrete electrodes with positions $\mathbf{x}_{j}$, the external work term is defined in terms of the local mechanical displacement, $\mathbf{u}_{k}=\mathbf{u}\left(\mathbf{x}_{k}, t\right)$, and the scalar electrical potential, $\varphi_{j}=\varphi\left(\mathbf{x}_{j}, t\right)$ :

$$
\delta W=\sum_{k=1}^{n f} \delta \mathbf{u}_{k} \mathbf{f}_{k}(t)-\sum_{j=1}^{n q} \delta \varphi_{j} q_{j}(t)
$$

The above definitions, as well as the constitutive relations of a piezoelectric material (see Eq. (13)), are used in conjunction with a variational approach to rewrite Eq. (23):

$$
\begin{aligned}
\int_{t 1}^{t 2} & {\left[\int_{V s} \rho_{s} \delta \dot{\mathbf{u}}^{t} \dot{\mathbf{u}} d V_{s}+\int_{V p} \rho_{p} \delta \dot{\mathbf{u}}^{t} \dot{\mathbf{u}} d V_{p}-\int_{V s} \delta \mathbf{S}^{t} \mathbf{c}_{s} \mathbf{S} d V_{s}-\int_{V p} \delta \mathbf{S}^{t} \mathbf{c}^{E} \mathbf{S} d V_{p}\right.} \\
& +\int_{V p} \delta \mathbf{S}^{t} \mathbf{e}^{t} \mathbf{E} d V_{p}+\int_{V p} \delta \mathbf{E}^{t} \mathbf{e} \mathbf{S} d V_{p}+\int_{V p} \delta \mathbf{E}^{t} \varepsilon^{S} \mathbf{E} d V_{p} \\
& \left.+\sum_{k=1}^{n f} \delta \mathbf{u}_{k} \mathbf{f}_{k}(t)-\sum_{j=1}^{n q} \delta \varphi_{j} q_{j}\right] d t=0
\end{aligned}
$$


Four basic assumptions are introduced: the Rayleigh-Ritz procedure, EulerBernoulli beam theory, and that the electrical field across the piezoelectric is constant. These assumptions are consistent with previous modeling efforts (e.g., $[36,37,65])$. In the Rayleigh-Ritz approach, the displacement of a structure can be written as the sum of $n r$ individual modes, $\psi_{r i}(\mathbf{x})$, multiplied by a mechanical temporal coordinate, $r_{i}(t)$, as in Eq. (29) [66]. For a beam in bending, only the transverse displacement is considered and the mode shape is a function only of the axial position, $x_{a}$, such that $\mathbf{u}(\mathbf{x}, t) \equiv w\left(x_{a}, t\right)$. Furthermore, the base excitation is assumed to be in the transverse direction as well. Similarly, the electric potential for each of the $n q$ electrode pairs can be written in terms of a potential distribution, $\psi_{v j}$, and the electrical temporal coordinate, $v_{j}(t)$, as in Eq. (30). Note that the interdigitated electrodes consist only of one electrode pair $(n q=1)$. The Euler-Bernoulli beam theory allows the axial strain in the beam to be written in terms of the beam displacement and the distance from the neutral axis, as in Eq. (31).

$$
\begin{aligned}
& \mathbf{u}(\mathbf{x}, t)=w\left(x_{a}, t\right)=\sum_{i=1}^{n r} \psi_{r i}\left(x_{a}\right) r_{i}(t)=\psi_{r}\left(x_{a}\right) \mathbf{r}(t) \\
& \varphi(\mathbf{x}, t)=\sum_{j=1}^{n q} \psi_{v j}(\mathbf{x}) v_{j}(t)=\psi_{v}(\mathbf{x}) \mathbf{v}(t) \\
& \mathbf{S}(\mathbf{x}, t)=-x_{t} \frac{\partial^{2} w\left(x_{a}, t\right)}{\partial x_{a}^{2}}=-x_{t} \boldsymbol{\psi}^{\prime \prime}{ }_{r} \mathbf{r}(t)
\end{aligned}
$$

Prime indicates the derivative to the axial position, $x_{a}$. The above assumptions allow Eq. (28) to be written in terms of mass, $\mathbf{M}$, stiffness, $\mathbf{K}$, coupling, $\boldsymbol{\Theta}$, and capacitive terms, $\mathbf{C}_{p}$, to obtain the governing equations in Eqs. (32-33). The coefficients are defined in Eqs. (34-37).

$$
\begin{aligned}
\mathbf{M} \ddot{\mathbf{r}}+\mathbf{K r}-\boldsymbol{\Theta} \mathbf{v}= & \sum_{k=1}^{n f} \boldsymbol{\psi}_{r}^{t}\left(x_{a k}\right) \cdot \mathrm{f}_{k}(t) \\
\boldsymbol{\Theta}^{t} \mathbf{r}+\mathbf{C}_{p} \mathbf{v}= & \sum_{j=1}^{n q} \boldsymbol{\psi}_{v}\left(\mathbf{x}_{j}\right) \cdot q_{j} \\
\mathbf{M}= & \int_{V s} \boldsymbol{\psi}_{r}^{t} \rho_{s} \boldsymbol{\psi}_{r} d V_{s}+\int_{V p} \boldsymbol{\psi}_{r}^{t} \rho_{p} \boldsymbol{\psi}_{r} d V_{p} \\
\mathbf{K}= & \int_{V s}\left(-x_{t} \boldsymbol{\psi}^{\prime \prime}{ }_{r}\right)^{t} \mathbf{c}_{s}\left(-x_{t} \psi^{\prime \prime}{ }_{r}\right) d V_{s} \\
& +\int_{V s}\left(-x_{t} \boldsymbol{\psi}^{\prime \prime}{ }_{r}\right)^{t} \mathbf{c}^{E}\left(-x_{t} \boldsymbol{\psi}^{\prime \prime}{ }_{r}\right) d V_{p}
\end{aligned}
$$




$$
\begin{aligned}
\boldsymbol{\Theta} & =\int_{V p}\left(-x_{t} \boldsymbol{\psi}^{\prime \prime}{ }_{r}\right)^{t} \mathbf{e}^{t}\left(-\nabla \cdot \boldsymbol{\psi}_{v}\right) d V_{p} \\
\mathbf{C}_{p} & =\int_{V s}\left(-\nabla \cdot \boldsymbol{\psi}_{v}\right)^{t} \varepsilon^{S}\left(-\nabla \cdot \boldsymbol{\psi}_{v}\right) d V_{p}
\end{aligned}
$$

The input to the system is a base excitation. The structure is discretized into $n f$ elements of length $\Delta x_{a}$ and the local inertial load is applied on the $k$ th element, or $\mathrm{f}_{k}=-m_{k} \Delta x_{a} \ddot{w}_{B}$. This results in $n f$ point loads. $m_{k}$ is the element mass per length. The loading is summated for all the elements. In the limit of $\Delta x_{a} \rightarrow d x_{a}$, the summation reduces to the integral over the structure length and a mass per length distribution is used, $m\left(x_{a}\right)$. For simplicity it has been assumed that the device is uniform in the axial direction, so that $m\left(x_{a}\right)=m=$ const . Substituting the forcing function into the right hand side of Eq. (32), the "forcing vector", $\mathbf{B}_{f}$, is defined.

$$
\mathbf{B}_{f}=-\int_{0}^{L} m\left(x_{a}\right) \boldsymbol{\psi}_{r}^{t} d x_{a}=-m \int_{0}^{L} \boldsymbol{\psi}_{r}^{t} d x_{a}
$$

The right hand side term of Eq. (33) reduces to a column vector of length $n q$ (the number of voltage modes) with element values $q_{t o t}$, where $q_{t o t}=\sum_{j=1}^{n q} q_{j}$. This equation can be differentiated with respect to time to obtain current. The current can be related to the voltage, assuming that the electrical loading is a resistor, $R_{l}$.

Mechanical damping is added through the addition of a damping matrix, C, to Eq. (32). When multiple modes are investigated, a proportional damping scheme is often used to ensure uncoupling of the equations during the modal analysis [66].

$$
\begin{aligned}
\mathbf{M} \ddot{\mathbf{r}}+\mathbf{C} \dot{\mathbf{r}}+\mathbf{K r}-\Theta \mathbf{v} & =\mathbf{B}_{f} \ddot{w}_{B} \\
\boldsymbol{\Theta}^{t} \dot{\mathbf{r}}+\mathbf{C}_{p} \dot{\mathbf{v}}+1 / R_{l} \mathbf{v} & =0
\end{aligned}
$$

It is important to note the similarities between the governing equations for the vibrating beam (Eqs. $(39,40))$ and the basic 1-D device (Eqs. $(15,16))$. When only a single beam mode is considered with one pair of electrodes, Eqs. $(39,40)$, reduce to two scalar equations. The damping scalar damping ensures that the equations uncouple automatically. The same optimization scheme in the 1$\mathrm{D}$ analysis can be used to find the maximum power developed, as well as the optimal load resistance for different driving frequencies. Lastly, the damping for a physical device is determined by the geometry and the operating environment (as discussed earlier). The damping is measured at the device natural frequency, which is fixed. As a consequence, the damping dependence on frequency need not be considered here if it can be assumed that the damping ratios at the resonance and anti-resonance frequencies are the same. 


\section{Modal Analysis: Cantilever Beam with a Mass at the Free End}

Since the target frequencies of the piezoelectric energy harvester under investigation are very low for MEMS-scale devices, it will be necessary to add a mass at the tip of the cantilever beam. The modal shapes and natural frequencies for a fixed-free cantilever beam are readily available in vibration texts (e.g., [66]), but the analysis with the addition of the mass is not as common, and will be covered briefly. This section is adapted from [67-69].

It is assumed that the center of gravity of the mass does not coincide with the end of the beam, $O$. Please refer to Fig. 13 for an illustration of the assumed beam configuration. The Euler-Bernoulli beam theory is used to determine the governing equations in terms of the mechanical displacement (Eq. (41)) and can be solved generally for the $N$ th mode, $N \in\{1, n r\}$ (Eq. (42)).

$$
\begin{gathered}
E I \psi_{r N}^{I V}-m \omega^{2} \psi_{r N}=0 \\
\psi_{r N}=c \sinh \lambda_{N} x_{a}+d \cosh \lambda_{N} x_{a}+e \sin \lambda_{N} x_{a}+f \cos \lambda_{N} x_{a}
\end{gathered}
$$

The arbitrary constants $(c, d, e$, and $f$ ) are solved using the boundary conditions of the beam with the mass. It is assumed that the both the beam and the proof mass are uniform in the axial direction with mass per lengths of $m$ and $m_{m}$, respectively. Using energy methods, it is possible to determine the boundary conditions at the point where the beam and the mass are connected, $w_{L}$ :

$$
\begin{gathered}
E I w_{L}^{\prime \prime}-\omega_{N}^{2} I_{o} w_{L}^{\prime}-\omega_{N}^{2} S_{0} w_{L}=0 \\
E I w_{L}^{\prime \prime \prime}+\omega_{N}^{2} M_{0} w_{L}+\omega_{N}^{2} S_{0} w_{L}^{\prime}=0
\end{gathered}
$$

where: $M_{0}=m_{m} L_{0}, S_{0}=M_{0} o_{x}, I_{0}=I_{y y}+M_{0}\left(o_{x}^{2}+o_{z}^{2}\right), E$ is the axial modulus of the beam, $I$ is the second moment of area of the beam, $I_{y y}$ is the moment of inertia of the proof mass around its center of gravity, and $\omega_{N}$ is the natural frequency of the beam. By defining $\bar{\lambda}_{N}=\lambda_{N} L, \bar{M}_{0}=M_{0} / m L, \bar{S}_{0}=S_{0} / m L^{2}$,
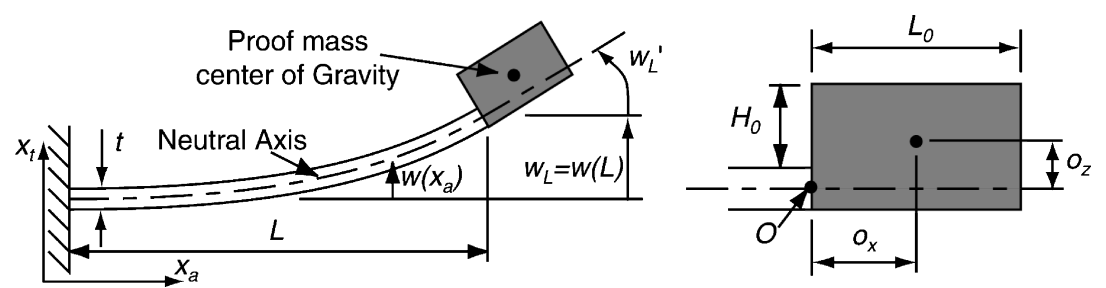

Figure 13. Beam with proof mass: modal analysis parameters. 
and $\bar{I}_{0}=I_{0} / m L^{3}$, the boundary conditions are used to obtain the matrix equation of the constants.

$$
\begin{aligned}
& {\left[\begin{array}{ll}
A_{11} & A_{12} \\
A_{21} & A_{22}
\end{array}\right]\left[\begin{array}{l}
e \\
f
\end{array}\right]=0 } \\
A_{11}= & \left(\sinh \bar{\lambda}_{N}+\sin \bar{\lambda}_{N}\right)+\bar{\lambda}_{N}^{3} \bar{I}_{0}\left(-\cosh \bar{\lambda}_{N}+\cos \bar{\lambda}_{N}\right) \\
& +\bar{\lambda}_{N}^{2} \bar{S}_{0}\left(-\sinh \bar{\lambda}_{N}+\sin \bar{\lambda}_{N}\right) \\
A_{12}= & \left(\cosh \bar{\lambda}_{N}+\cos \bar{\lambda}_{N}\right)+\bar{\lambda}_{N}^{3} \bar{I}_{0}\left(-\sinh \bar{\lambda}_{N}-\sin \bar{\lambda}_{N}\right) \\
& +\bar{\lambda}_{N}^{2} \bar{S}_{0}\left(-\cosh \bar{\lambda}_{N}+\cos \bar{\lambda}_{N}\right) \\
A_{21}= & \left(\cosh \bar{\lambda}_{N}+\cos \bar{\lambda}_{N}\right)+\bar{\lambda}_{N} \bar{M}_{0}\left(\sinh \bar{\lambda}_{N}-\sin \bar{\lambda}_{N}\right) \\
& +\bar{\lambda}_{N}^{2} \bar{S}_{0}\left(\cosh \bar{\lambda}_{N}-\cos \bar{\lambda}_{N}\right) \\
A_{22}= & \left(\sinh \bar{\lambda}_{N}-\sin \bar{\lambda}_{N}\right)+\bar{\lambda}_{N} \bar{M}_{0}\left(\cosh \bar{\lambda}_{N}-\cos \bar{\lambda}_{N}\right) \\
& +\bar{\lambda}_{N}^{2} \bar{S}_{0}\left(\sinh \bar{\lambda}_{N}+\sin \bar{\lambda}_{N}\right)
\end{aligned}
$$

The mode resonance frequencies are obtained by solving for $\bar{\lambda}_{N}$ such that $\left|\begin{array}{ll}A_{11} & A_{12} \\ A_{21} & A_{22}\end{array}\right|=0$. Successive values of $\bar{\lambda}_{N}$ correspond to the modes of the beam and the natural frequency of each mode can be determined with: $\omega_{N}^{2}=\bar{\lambda}_{N}^{2} \sqrt{\frac{E I}{m L^{4}}}$. The solution, Eq. (42), can be written in terms of a single arbitrary constant, say $f$ :

$$
\psi_{r N}=f\left[\left(\cosh \lambda_{N} x_{a}-\cos \lambda_{N} x_{a}\right)-A_{12} / A_{11}\left(\sinh \lambda_{N} x_{a}-\sin \lambda_{N} x_{a}\right)\right]
$$

The effective mass of the structure is obtained from the Lagrange equations of motion and is given in Eq. (51). Note that Eq. (51) replaces Eq. (34) when a proof mass is added to a cantilever beam.

$$
\begin{aligned}
\mathbf{M}= & \int_{V s} \boldsymbol{\psi}_{r}^{t} \rho_{s} \boldsymbol{\psi}_{r} d V_{s}+\int_{V p} \boldsymbol{\psi}_{r}^{t} \rho_{p} \boldsymbol{\psi}_{r} d V_{p}+M_{0}\left(\boldsymbol{\psi}_{r}(L)\right)^{t}\left(\boldsymbol{\psi}_{r}(L)\right) \\
& +2 S_{0}\left(\boldsymbol{\psi}_{r}(L)\right)^{t} \boldsymbol{\psi}^{\prime}{ }_{r}(L)+I_{0}\left(\boldsymbol{\psi}_{r}^{\prime}(L)\right)^{t}\left(\boldsymbol{\psi}_{r}^{\prime}(L)\right)
\end{aligned}
$$

Lastly, the external work term needs to be re-evaluated to include the inertial loading due to the proof mass at the beam tip. In Eq. (38), the forcing vector, $\mathbf{B}_{f}$, was defined to account for the inertial loading due to a base excitation. It was previously assumed (for simplicity) that the device is uniform in the axial direction. However, the device now consists of two separate sections, the uniform beam and uniform proof mass. Both contribute to the inertial loading of the device. The proof mass displacement is calculated in terms of the displacement and rotation of the tip of the beam. A forcing function is defined in 
terms of the mass per length of the proof mass, $m_{m}$, and two additional terms are calculated to make up the modified input matrix:

$$
\begin{aligned}
\mathbf{B}_{f}= & -\left(m \int_{0}^{L L}\left\{\boldsymbol{\psi}_{r}\left(x_{a}\right)\right\}^{t} d x_{a}+m_{m} \int_{L}^{L+L_{0}}\left\{\boldsymbol{\psi}_{r}(L)\right\}^{t} d x_{a}\right. \\
& \left.+m_{m} \int_{L}^{L+L_{0}}\left\{\boldsymbol{\psi}_{r}^{\prime}(L) x_{a}\right\}^{t} d x_{a}\right)
\end{aligned}
$$

\section{$\{$ 3-1 $\}$ Mode vs. $\{$ 3-3 $\}$ Mode of Operation}

For piezoelectric elements, the longitudinal piezoelectric effect can be much larger than the traverse effect $\left(d_{33} / d_{31} \sim 2.4\right.$ for most piezoelectric ceramics, see for example [70-72]). For this reason it is desirable to operate the device in the $\{3-3\}$, or longitudinal, mode. Longitudinal mode operation occurs when the electric field and the strain direction coincide. Refer to Fig. 14 for an illustration of the two configurations. Conventionally, the electrodes are placed on the top and bottom surfaces of the piezoelectric element. The electric field is through the thickness of the piezoelectric element, while the strain is in the axial direction, and the transverse, or $\{3-1\}$, mode is utilized. Through the use of interdigitated electrodes, a large component of the electric field can be in the axial direction. This configuration has been utilized in the past [70-74].

\section{Conventional Piezoelectric Energy Harvester Utilizing the $\{3-1\}$ Mode}

The conventional cantilever configuration piezoelectric energy harvester model ( $\{3-1\}$ mode) is presented before considering the $\{3-3\}$ interdigitated unimorph cantilever. Please refer to Fig. 14 for an illustration of the configuration, as well as the definition of the parameters. Assuming plane stress for a plate (refer to [59] for a detailed description of plane stress and strain constitutive reductions), the constitutive relations (Eq. (13)) can be simplified by taking

$\{3-1\}$ Mode

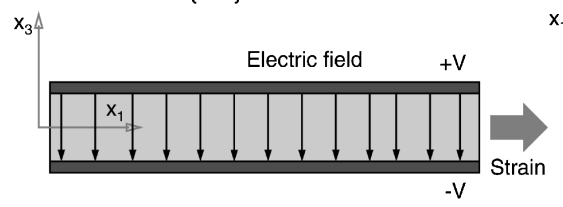

$\{3-3\}$ Mode

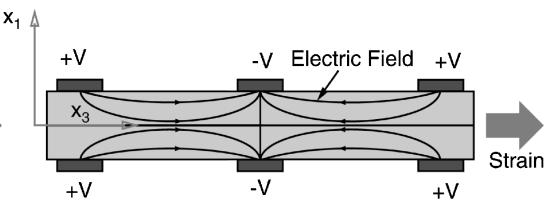

Figure 14. $\{3-1\}$ vs. $\{3-3\}$ modes of operation. 
$T_{3}=T_{4}=T_{5}=0$ :

$$
\left[\begin{array}{c}
T_{1} \\
T_{2} \\
T_{6} \\
D_{3}
\end{array}\right]=\left[\begin{array}{cccc}
c_{11}^{E^{*}} & c_{12}^{E^{*}} & 0 & -e_{31}^{*} \\
c_{12}^{E^{*}} & c_{11}^{E^{*}} & 0 & -e_{31}^{*} \\
0 & 0 & c_{66}^{E^{*}} & 0 \\
e_{31}^{*} & e_{31}^{*} & 0 & \varepsilon_{33}^{S^{*}}
\end{array}\right]
$$

For the plate which bends only in one direction, the constitutive equations can be further reduced by taking $S_{2}=S_{6}=0$ :

$$
\left[\begin{array}{l}
T_{1} \\
D_{3}
\end{array}\right]=\left[\begin{array}{cc}
c_{11}^{E^{*}} & -e_{31}^{*} \\
e_{31}^{*} & \varepsilon_{33}^{S^{*}}
\end{array}\right]\left[\begin{array}{l}
S_{1} \\
E_{3}
\end{array}\right]
$$

It is important to note that due to the plane stress assumption, the piezoelectric constants in Eq. (53) are not equal to the fully 3-D constants. These constants have to be determined from the compliance form of the constitutive relations (i.e., with stress as the independent field variable and in terms of the compliance, $\mathbf{s}^{E}$ ), resulting in:

$$
\begin{aligned}
c_{11}^{E^{*}} & =\frac{s_{11}^{E}}{\left(s_{11}^{E}\right)^{2}-\left(s_{12}^{E}\right)^{2}} \\
e_{31}^{*} & =\frac{s_{11}^{E} d_{31}-s_{12}^{E} d_{31}}{\left(s_{11}^{E}\right)^{2}-\left(s_{12}^{E}\right)^{2}} \\
\varepsilon_{33}^{S^{*}} & =\varepsilon_{33}^{T}-\frac{2 d_{31}^{2}\left(s_{11}^{E}-s_{12}^{E}\right)}{\left(s_{11}^{E}\right)^{2}-\left(s_{12}^{E}\right)^{2}}
\end{aligned}
$$

The following electric potential distribution was assumed to give a constant electric field through the thickness of the piezoelectric element. The potential varies from +1 at top electrode to -1 at the bottom electrode. Taking only the first mode of the beam, the functions $\psi_{r}$ and $\psi_{v}$ in Eq. (29-30) become:

$$
\begin{aligned}
& \psi_{v}=\psi_{v 1}=\frac{\frac{t_{p}}{2}-\left(x_{3}-\frac{t_{p}}{2}\right)}{\frac{t_{p}}{2}} \\
& \psi_{r}=\psi_{r 1}=f\left[\left(\cosh \lambda_{1} x_{1}-\cos \lambda_{1} x_{1}\right)-A_{12} / A_{11}\left(\sinh \lambda_{1} x_{1}-\sin \lambda_{1} x_{1}\right)\right]
\end{aligned}
$$

With these assumed modes, the system response is calculated in closed form since the governing equations are scalar. First, the governing equation 
(Eq. (39)) is written in an alternative form by dividing through by $M$ and making use of the definitions $\omega_{1}=\sqrt{K / M}$ and $\zeta_{m}=C / M 2 \omega_{1}$.

$$
\begin{gathered}
\ddot{r}+2 \zeta_{m} \omega_{1} \dot{r}+\omega_{1}^{2} r-\Theta / M v=B_{\mathrm{f}} \ddot{w}_{B} / M \\
\Theta \dot{r}+C_{p} \dot{v}+1 / R_{l} v=0
\end{gathered}
$$

The dimensionless factors $R_{e}=\omega_{1} R_{l} C_{p}$ and $\Omega=\omega / \omega_{1}$ are introduced, where $\omega$ is the base input frequency and the system response is calculated. The resulting equations are similar to those derived for the basic 1-D piezoelectric model.

$\left|\frac{r_{x}}{\ddot{w}_{B}}\right|=\frac{B_{\mathrm{f}} / K \sqrt{1+\left(R_{e} \Omega\right)^{2}}}{\sqrt{\left[1-\left(1+2 \zeta_{m} R_{e}\right) \Omega^{2}\right]^{2}+\left[\left(2 \zeta_{m}+\left\{1+\Theta^{2} / K C_{p}\right\} R_{e}\right) \Omega-R_{e} \Omega^{3}\right]^{2}}}$

$\left|\frac{v}{\ddot{w}_{B}}\right|=\frac{B_{\mathrm{f}} / K C_{p} R_{e} \Theta \Omega}{\sqrt{\left[1-\left(1+2 \zeta_{m} R_{e}\right) \Omega^{2}\right]^{2}+\left[\left(2 \zeta_{m}+\left\{1+\Theta^{2} / K C_{p}\right\} R_{e}\right) \Omega-R_{e} \Omega^{3}\right]^{2}}}$

$\left|\frac{P_{\text {out }}}{\left(\ddot{w}_{B}\right)^{2}}\right|=\frac{\left(B_{\mathrm{f}} / K \Theta \Omega\right)^{2} \omega_{1} 1 / C_{p} R_{e}}{\left[1-\left(1+2 \zeta_{m} R_{e}\right) \Omega^{2}\right]^{2}+\left[\left(2 \zeta_{m}+\left\{1+\Theta^{2} / K C_{p}\right\} R_{e}\right) \Omega-R_{e} \Omega^{3}\right]^{2}}$

Again, the system can be analyzed at short and open circuit conditions by letting the load resistance tend to zero and infinity respectively. Two frequency ratios are obtained, which correspond to the resonance (short circuit) and anti-resonance (open circuit) frequencies of the piezoelectric element.

$$
\begin{aligned}
& \Omega_{s c}=1 \\
& \Omega_{o c}=\sqrt{1+\Theta^{2} / K C_{p}}
\end{aligned}
$$

The power can be optimized with respect to the load resistance to obtain an optimal electrical load:

$$
R_{e, o p t}=\sqrt{\frac{\Omega^{4}+\left(4 \zeta_{m}^{2}-2\right) \Omega^{2}+1}{\Omega^{6}+\left(4 \zeta_{m}^{2}-2\left(1+\Theta^{2} / K C_{p}\right)\right) \Omega^{4}+\left(1+\Theta^{2} / K C_{p}\right)^{2} \Omega^{2}}}
$$


Comparing these results to the 1-D model, we identify similar behavior for the cantilever configuration as for the basic 1-D configuration. The antiresonance is determined by the coupling term $\Theta^{2} / K C_{p}$. Since the structure is made up of both piezoelectric- and non-piezoelectric layers, this term does not in general correspond to the coupling coefficient as defined before (section on 1-D model), but reduces to this coefficient for a purely piezoelectric beam.

The model was applied to published experiments and analytical results obtained for a macro-scale bimorph [36] using a Quick Pack QP40N actuator from Midé Technology Corporation. The Quick Pack is a built-up plate device consisting of two piezoelectric element pairs $\left(t_{p}=0.254 \mathrm{~mm}\right)$ embedded in a Kapton and epoxy matrix $\left(t_{s}=0.254 \mathrm{~mm}\right)$ with overall dimensions of $100.6 \mathrm{~mm} \times 25.4 \mathrm{~mm} \times 0.762 \mathrm{~mm}(L \times b \times t)$. No mass was present at the end of the plate and the plate was clamped $8 \mathrm{~mm}$ from the end to have an effective length of $92.6 \mathrm{~mm}$. PZT-5A properties and a mechanical damping ratio of $\zeta_{m}=0.01$ were assumed for the analysis. Base acceleration is a key assumption for the power generated, as is the bending stiffness for the natural frequency estimation. The input vibration amplitude was not specified, so a base acceleration of $3 \mathrm{~m} / \mathrm{s}^{2}$ was used, which was found to be within static failure limits for the piezoelectric material. Effective bending stiffness was calculated from the published experimental first resonance frequency of around $33 \mathrm{~Hz}$ as 23.553 $10^{-3} \mathrm{Nm}^{2}$.

As an approximation, the device is modeled with a single electrode, spanning the length of the plate and with a width of $20.6 \mathrm{~mm}$. This will cause the model to over-predict the power performance slightly. The forms of $\psi_{r}$ and $\psi_{v}$ are given in Eqs. (57-58), assuming constant electric potential through the thickness of the piezoelectric element and one beam mode. With these approximations, the predicted performance was compared to the published results. Please refer to Table 3 for selected values. The published and predicted currents and current trends compare well and help to validate the model.

The analysis was extended to include the resonance response of the device, and the two power-optimal operating points. The power vs. frequency ratio is plotted, with varying load resistances in Fig. 15. The shift in operating

Table 3

\{3-1\} Bimorph beam comparison: measured and predicted results

\begin{tabular}{lccccr}
\hline $\begin{array}{l}\text { Frequency } \\
{[\mathrm{Hz}]}\end{array}$ & $\begin{array}{c}\text { Load } \\
\text { resistance } \\
{[\Omega]}\end{array}$ & $\begin{array}{c}\text { Predicted } \\
\text { current } \\
{[\mathrm{mA}], \text { Ref. [36] }}\end{array}$ & $\begin{array}{c}\text { Measured } \\
\text { current } \\
{[\mathrm{mA}], \text { Ref. [36] }}\end{array}$ & $\begin{array}{c}\text { Predicted } \\
\text { current } \\
{[\mathrm{mA}]}\end{array}$ & $\begin{array}{c}\text { Percent } \\
\text { error }\end{array}$ \\
\hline 25 & 100 & 0.101 & 0.104 & 0.111 & 6.73 \\
25 & 10,000 & 0.105 & 0.106 & 0.098 & -7.55 \\
30 & 100 & 0.360 & 0.345 & 0.343 & -0.58 \\
30 & 10,000 & 0.295 & 0.300 & 0.217 & -27.7 \\
\hline
\end{tabular}




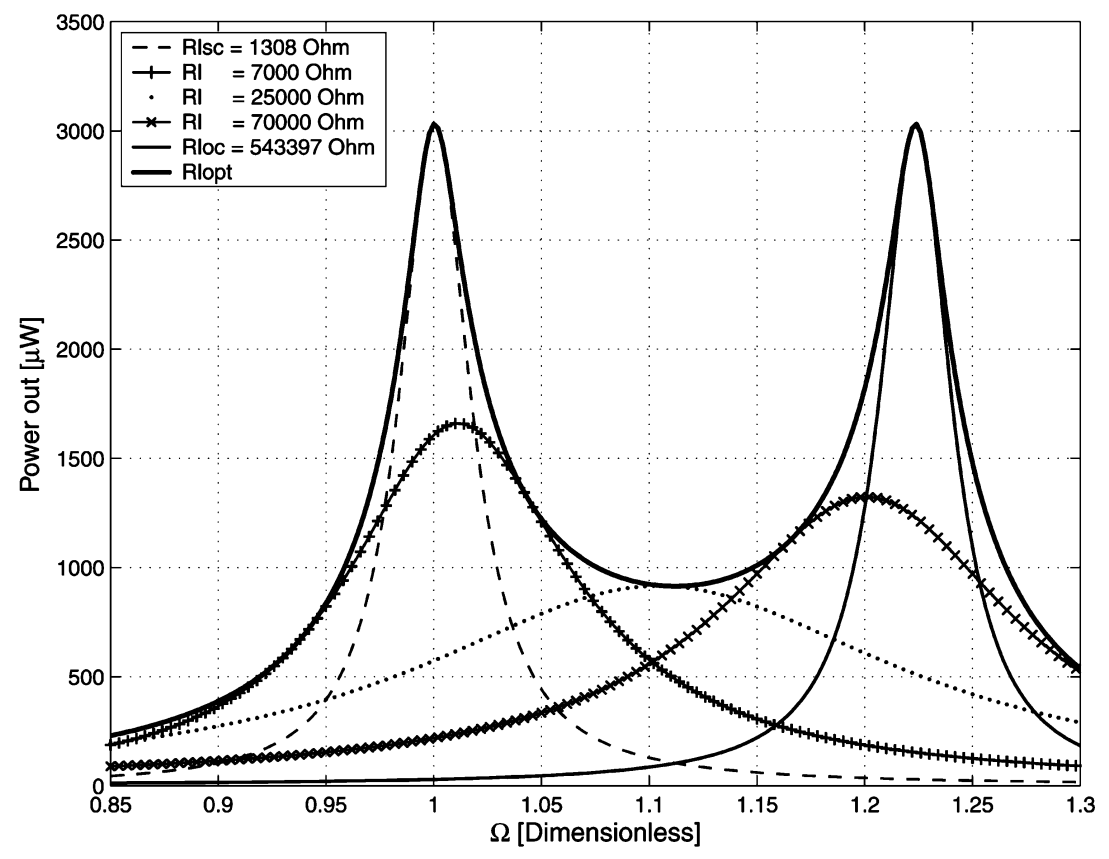

Figure 15. Power vs. normalized frequency with varying electrical load resistance for $\{3-1\}$ bimorph beam in [36].

frequency with the increasing load resistance from the resonance to the antiresonance frequency is clearly visible as the shift in frequency ratio is $\sim 12.2 \%$, corresponding to $\sim 7.5 \mathrm{~Hz}$. This frequency shift is very pronounced because the piezoelectric element's contribution to the overall stiffness of the device is large. The maximum power predicted is around 3,000 $\mu \mathrm{W}$, as compared to the maximum power measured of $900 \mu \mathrm{W}$ (for the $30 \mathrm{~Hz}, 100,000 \mathrm{Ohm}$ resistive load combination). This power prediction emphasizes the importance of selecting the correct operating point, as well aligning the device natural frequency with the dominant vibration component of the source. Please refer to Table 4 for a summary of the predicted performance.

A second bimorph device was modeled and compared to published, simulated results [5]. The device consisted of two piezoelectric elements with no structural layer and a mass at the tip. The overall beam dimensions are $16.2 \mathrm{~mm} \times 3 \mathrm{~mm} \times 0.73 \mathrm{~mm}(L \times b \times t)$ and the mass has dimensions 17.1 $\mathrm{mm} \times 3 \mathrm{~mm} \times 5 \mathrm{~mm}\left(L_{0} \times b_{0} \times H_{0}\right)$. No material properties are given for the device. Assuming PZT-5A and steel (for the mass) material properties, resonance and anti-resonance frequencies of $118 \mathrm{~Hz}$ and $134 \mathrm{~Hz}$ respectively are predicted, compared to the $120 \mathrm{~Hz}$ specified. With an input acceleration of 
Table 4

Predicted performance for $\{3-1\}$ bimorph beam modeled in Fig. 15

\begin{tabular}{lcc}
\hline & \multicolumn{2}{c}{$3-1$} model analysis \\
\cline { 2 - 3 } Parameter & Short circuit & Open circuit \\
\hline Maximum power out $[\mu \mathrm{W}]$ & 3,030 & 3,030 \\
Power density $\left[\mu \mathrm{W} / \mathrm{cm}^{3}\right]^{\dagger}$ & 200 & 238 \\
Natural frequency $[\mathrm{Hz}]$ & 32.8 & 40.1 \\
Optimal resistance $[\mathrm{kOhm}]$ & 1.31 & 543 \\
Tip displacement of beam $[\mathrm{mm}]$ & 2.84 & 2.32 \\
Maximum strain $[\mu \mathrm{m} / \mathrm{m}]$ & 443 & 362 \\
Voltage $[\mathrm{V}]$ & 2.0 & 40.5 \\
Current $[\mathrm{mA}]$ & 1.52 & 0.074 \\
\hline
\end{tabular}

${ }^{\dagger}$ Device volume is approximated as $\mathrm{L} \times \mathrm{b} \times(2\{$ tip displacement $\}+$ thickness).

$2.5 \mathrm{~m} / \mathrm{s}^{2}$, a power of $785 \mu \mathrm{W}$ is predicted, compared to the published power prediction of $242 \mu \mathrm{W}$. The deviation is ascribed to the uncertainty in material properties and a more rigorous treatment of the beam and mass mode shapes in the current analysis.

\section{Piezoelectric Energy Harvester Utilizing the $\{3-3\}$ Effect}

Operating the piezoelectric element in the $\{3-3\}$ actuation mode is advantageous, as better coupling between the mechanical and electrical domain is possible. An approximate model for the interdigitated electrode-configuration has been adopted. It is assumed that the region of the piezoelectric element under the electrode is electrically inactive, whereas the section between the electrodes utilizes the full $\{3-3\}$ effect. These are first approximations, since the electric field is not completely in the axial direction through the thickness of the piezoelectric element, nor is the section of piezoelectric element under the electrode completely inactive. These effects compensate for each other somewhat. Please refer to Fig. 16 for the geometry of the approximate model.

Again, plane stress for a plate is assumed, and with the plate geometry defined in Fig. 14, the constitutive relations for the active sections in Fig. 16 can be reduced to:

$$
\left[\begin{array}{l}
T_{3} \\
D_{3}
\end{array}\right]=\left[\begin{array}{cc}
c_{33}^{E^{* *}} & -e_{33}^{* *} \\
e_{33}^{* *} & \varepsilon_{33}^{S^{* *}}
\end{array}\right]\left[\begin{array}{l}
S_{3} \\
E_{3}
\end{array}\right]
$$



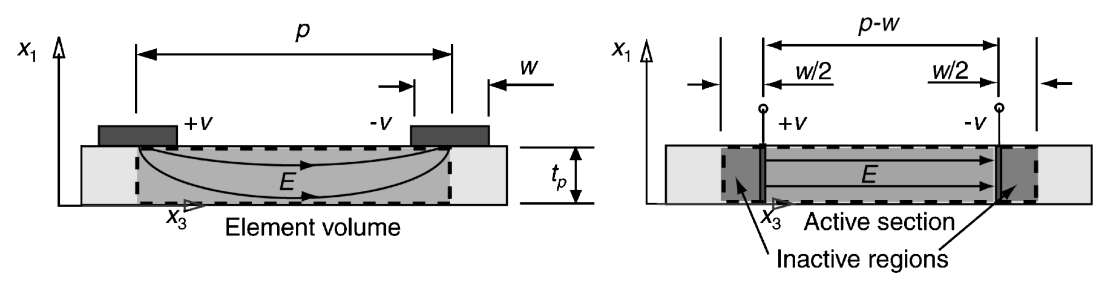

Figure 16. Interdigitated electrode configuration (left) and model approximation (right).

Due to the plane stress assumption the piezoelectric constants will not be equal to any of the fully 3-D constants, but are defined as follows:

$$
\begin{aligned}
c_{33}^{E^{* *}} & =\frac{s_{11}^{E}}{s_{11}^{E} s_{33}^{E}-\left(s_{13}^{E}\right)^{2}} \\
e_{33}^{* *} & =\frac{s_{11}^{E} d_{33}-s_{13}^{E} d_{31}}{s_{11}^{E} s_{33}^{E}-\left(s_{13}^{E}\right)^{2}} \\
\varepsilon_{33}^{S^{* *}} & =\varepsilon_{33}^{T}-\frac{1}{s_{11}^{E} s_{33}^{E}-\left(s_{13}^{E}\right)^{2}}\left(s_{33}^{E} d_{31}^{2}-2 s_{13}^{E} d_{31} d_{33}+s_{11}^{E} d_{33}^{2}\right)
\end{aligned}
$$

With the approximation that the region of piezoelectric element beneath the electrode is inactive, while the section of piezoelectric element between the electrodes experiences coupling through the full longitudinal mode, we can specify an electrical potential distribution. To have a constant electric field between the electrodes, the potential distribution needs to vary from +1 at the electrode on the left to -1 at the electrode on the right in Fig. 16. Again only the first beam mode is considered.

$$
\begin{aligned}
& \boldsymbol{\psi}_{v}=\boldsymbol{\psi}_{v 1}=\frac{\frac{p-w}{2}-\left(x_{3}-\frac{p-w}{2}\right)}{\frac{p-w}{2}} \\
& \boldsymbol{\psi}_{r}=\boldsymbol{\psi}_{r 1}=f\left[\left(\cosh \lambda_{1} x_{3}-\cos \lambda_{1} x_{3}\right)-A_{12} / A_{11}\left(\sinh \lambda_{1} x_{3}-\sin \lambda_{1} x_{3}\right)\right]
\end{aligned}
$$

It is important to note that although the device is made up of a number of separate piezoelectric control volumes, there is only one electrode pair and the voltage across all the elements will be the same. Since the strain varies along the length of the of the beam, different amounts of charge will be generated in each element and the charge sums to give the total charge output of the device. Using a similar analysis as in the previous section, the same governing equation (Eq. (39)) is obtained. Only the coefficients of the equation will differ (due to the $\{3-3\}$ mode) and the same optimization scheme can be employed. 
To validate the model, a prototype device, previously built in our group [51, 61], was modeled. Please refer to Fig. 3 for the device configuration. The plate is $170 \mu \mathrm{m}$ long, $261 \mu \mathrm{m}$ wide and is made up of 5 layers: $\mathrm{SiN}_{x}(0.4 \mu \mathrm{m}), \mathrm{SiO}_{2}$ $(0.1 \mu \mathrm{m}), \mathrm{ZrO}_{2}(0.05 \mu \mathrm{m}), \mathrm{PZT}(0.48 \mu \mathrm{m})$, and the electrode (Ti $0.02 \mu \mathrm{m}$ and Pt $0.2 \mu \mathrm{m})$. A proof mass of SU-8 was added at the tip of the plate and has the following dimensions: $20 \mu \mathrm{m} \times 261 \mu \mathrm{m} \times 50 \mu \mathrm{m}\left(L_{0} \times b_{0} \times H_{0}\right)$. See Fig. 13 for the mass parameter definitions. A single interdigitated electrode pair was deposited on the top surface with a pitch, $\mathrm{p}$, of $\sim 8 \mu \mathrm{m}$ and the electrode arms width, w, was $4 \mu \mathrm{m}$ (see Fig. 16 for the parameter definitions). An effective electrode area of 50\% was used for the mass calculation. For the contribution of the electrodes to the stiffness of the plate, it was assumed that the arms of the electrode will affect the bending stiffness very little and only the connects (along the length of the plate near the plate width free edges) was used in the calculation with an effective width of $10 \%$. The plate tip displacement was measured at short circuit conditions and this value was used to estimate the mechanical damping ratio for the structure: $\zeta_{m}=0.0056$. Lastly, PZT material properties were measured: $d_{33}=20010^{-12} \mathrm{~m} / \mathrm{V}, \varepsilon_{33}^{T}=1200 \varepsilon_{0}$. A modulus of $c_{33}^{E}=63 \mathrm{GPa}$ is prescribed, but using PZT-5A compliance properties and the effective piezoelectric constants and stiffness (Eq. (67)), a modulus of $c_{33}^{E}=$ $64 \mathrm{GPa}$ is calculated. For $\mathrm{d}_{31}$ a value was calculated based on the ratio of $d_{31} / d_{33}$ for PZT-5A. The value used in the analysis is: $d_{31}=-91.710^{-12} \mathrm{~m} / \mathrm{V}$.

A resonance frequency of $13.7 \mathrm{kHz}$ was measured (at open circuit conditions), but was found to shift to around $13.9 \mathrm{kHz}$. With the model, a natural frequency of $12.66 \mathrm{kHz}$ is predicted for short circuit conditions, using the prescribed parameters. The deviation of the model from the measurement is ascribed to the uncertainty of material properties for the layers - including the value for the PZT modulus (values of $c_{33}^{E}$ for PZT-5A vary from $53 \mathrm{GPa}$ to $111 \mathrm{GPa}$ for different configurations). When the modulus is adjusted to $c_{33}^{E}=$ $77 \mathrm{GPa}$, a resonance frequency of $13.7 \mathrm{kHz}$ is predicted for short circuit conditions. At open circuit conditions, the anti-resonance frequency of $14.0 \mathrm{kHz}$ is predicted. The predicted shift in natural frequency corresponds extremely well with the $\sim 200 \mathrm{~Hz}$ shift in the measured natural frequency. A modulus of $64 \mathrm{GPa}$ and a reference base input of $14 \mathrm{~nm}$ at $13.7 \mathrm{kHz}$, giving a base acceleration of $103.7 \mathrm{~m} / \mathrm{s}^{2}$, were used. The displacement of the center of the mass of $1.75 \mu \mathrm{m}$ was predicted at short circuit conditions, compared with the measured result of $1.85 \mu \mathrm{m}$. The electrical performance measured cannot be modeled currently as the rectifier and storage electronics have not been incorporated in the model. This will be accomplished in future work.

\section{PRELIMINARY DESIGN: LOW-LEVEL MPVEH}

A preliminary design of a MEMS-scale low level vibration energy harvester using the developed models was undertaken, but not optimized. The fabrication 
process for the device has been established, placing limitations on the device geometry and size. The device structure is outlined with the proposed fabrication sequence. Next, the parameters for the preliminary design are presented, and finally the performance of the device is predicted.

The fabrication for a prototype device is based on the sequence developed in house [51, 61]. It is based on a 3-mask, 5-layer design. The first layer is a membrane layer consisting of Plasma-Enhanced Chemical Vapor Deposition (PECVD) $\mathrm{SiN}_{x}$ and $\mathrm{SiO}_{2}$. The thickness ratio of the materials is varied to control the residual stress and initial curvature of the structure. The second layer is a buffer layer of $\mathrm{ZrO}_{2}$ and is deposited with a spin-on process. The layer is necessary to prevent charge diffusion from the piezoelectric element into the $\mathrm{SiN}_{\mathrm{x}} / \mathrm{SiO}_{2}$ substrate. Since the $\mathrm{ZrO}_{2}$ does not adhere well to the $\mathrm{SiN}_{x}$ the $\mathrm{SiO}_{2}$ layer is a necessary interface. Third, a PZT layer is deposited with a spin-on process developed by Mitsubishi Materials Company. The first three layers are patterned with the first mask, using reactive-ion etching (RIE). The electrode layers ( $\mathrm{Ti}$ and $\mathrm{Pt}$ ) are deposited with an electron-beam evaporation process. The electrodes are patterned with the second mask and a lift-off process. The last layer is an SU-8 proof mass, which is deposited with a spin-on process and is patterned with the third mask. An isotropic $\mathrm{XeF}_{2}$-vapor etch is used to release the structure. The isotropic etch will lengthen the structure since the etch will form an undercut in the substrate, which needs to be accounted for.

The fabrication process limits the total length dimension to $<1 \mathrm{~mm}$, and the total beam thickness to $<2 \mu \mathrm{m}$. The design parameters are given in Table 5 , followed by the predicted performance for a $4.2 \mathrm{~m} / \mathrm{s}^{2}, 150 \mathrm{~Hz}$ vibration input. This input is the measured vibrations from a microwave oven side panel (refer to Table 2). A promising $28.2 \mu \mathrm{W} / \mathrm{cm}^{3}$ from the low-level MPVEH is predicted for the non-optimal design.

\section{FUTURE WORK}

An effective power normalization scheme is required in order to compare energy harvester devices of different sizes and with different vibration inputs to estimate efficiencies. Strain cancellation due to multiple input vibration components will be investigated further with the objective of defining an optimal electrode length. The efficiency of energy conversion from mechanical vibration energy to electrical energy will be investigated to determine a minimum vibration level required for positive energy harvesting given a known MPVEH performance.

A prototype device is to be built and tested under controlled conditions in order to further validate the presented models. An improved design for a MEMS-scale piezoelectric beam energy harvester will be performed based on the preliminary design performed herein and the prototype built and tested. Design schemes for achieving more compliant structures, in order to eliminate the proof mass, will be investigated. Lastly, the system level design of 
Table 5

MPVEH preliminary design: device parameters and predicted performance

\begin{tabular}{lclc}
\hline Device parameters & \multicolumn{3}{c}{ Predicted performance } \\
\hline Length, $\mathrm{L}[\mu \mathrm{m}]$ & 1,000 & Power extracted $[\mu \mathrm{W}]$ & 0.031 \\
Width, $\mathrm{b}[\mu \mathrm{m}]$ & 500 & Power density $\left[\mu \mathrm{W} / \mathrm{cm}^{3}\right]$ & 28.2 \\
Structural layer & $0.15 / 0.15 / 0.05$ & Short Circuit condition \\
$\quad\left(\mathrm{SiN}_{\mathrm{x}} / \mathrm{SiO}_{2} / \mathrm{ZrO}_{2}\right)[\mu \mathrm{m}]$ & & & \\
Piezoelectric layer $[\mu \mathrm{m}]$ & 0.5 & Resonance frequency $[\mathrm{Hz}]$ & 149.3 \\
Electrode layer $(\mathrm{Ti} / \mathrm{Pt})[\mu \mathrm{m}]$ & $0.02 / 0.2$ & Strain $[\mu \mathrm{m} / \mathrm{m}]$ & 767 \\
Electrode arm width, $\mathrm{w}[\mu \mathrm{m}]$ & 4 & Tip displacement $[\mu \mathrm{m}]$ & 445 \\
Electrode pitch, $\mathrm{p}[\mu \mathrm{m}]$ & 20 & Voltage $[\mathrm{V}]$ & 0.553 \\
Electrode overlap, $\mathrm{l}_{\text {overlap }}[\mu \mathrm{m}]$ & 476 & Optimal resistance $[\mathrm{M} \Omega]$ & 10.2 \\
Proof mass length, $\mathrm{L}_{0}[\mu \mathrm{m}]$ & 200 & Open Circuit condition \\
Proof mass width, $\mathrm{b}_{0}[\mu \mathrm{m}]$ & 1,000 & Anti-resonance & 152.0 \\
& & frequency $[\mathrm{Hz}]$ & \\
Proof mass height, $\mathrm{H}_{0}[\mu \mathrm{m}]$ & 50 & Strain $[\mu \mathrm{m} / \mathrm{m}]$ & 756 \\
Device total volume $\left[\mathrm{cm}^{3}\right]^{\dagger}$ & $1.1 \times 10^{-3}$ & Tip displacement $[\mu \mathrm{m}]$ & 440 \\
Mechanical damping ratio & 0.0056 & Voltage $[\mathrm{V}]$ & 1.88 \\
Base acceleration $\left[\mathrm{m} / \mathrm{s}^{2}\right]$ & 4.2 & Optimal resistance $[\mathrm{M} \Omega]$ & 118 \\
\hline
\end{tabular}

${ }^{\dagger}$ Device volume is approximated as $\left(\mathrm{L}+\mathrm{L}_{0}\right) \times\left(\mathrm{b}_{0}\right) \times(2\{$ maximum tip displacement $\left.\}+\mathrm{H}_{0}\right)$.

a MPVEH will be investigated, including incorporation of optimized power harvester electronics and storage.

\section{CONCLUSIONS AND RECOMMENDATIONS}

Design considerations related to the mechanical performance of piezoelectric energy harvesters were presented. It has been recommended that the following information be made available in future publications to facilitate a relative comparison of devices: device size (including and excluding the power electronics), the maximum tip displacement at maximum power output (to define the operating volume as in this work), the mechanical damping ratio, the electrical load, the device mass, and the input vibration characteristics (frequency and magnitude).

Ambient vibration energy sources were characterized and it was concluded that significant power is present in the range from $100-300 \mathrm{~Hz}$ for a number of ambient sources. An interpretation scheme, based on a general energy harvester model, was presented for vibration peak selection. It was shown that the peak selection scheme differs for macro- and micro-scale beam devices and is further influenced by the operating conditions for the micro-scale device (e.g., 
atmospheric vs. vacuum conditions) since the dominant damping components are different at the micro- vs. macro-scale.

A basic, fully coupled electromechanical model was developed to analyze the response of a piezoelectric energy harvester. The system was optimized for maximum power extraction. Two optimums for power extraction are identified, corresponding to the resonance and anti-resonance frequencies of the device. The shift in device natural frequency from short- to open-circuit conditions is more pronounced for micro-scale devices since the piezoelectric element's contribution to the overall structural stiffness is larger than for macro-scale devices. For macro-scale devices the effect is normally negligible. To date, all optimization schemes have neglected the anti-resonance operating point by assuming that the optimal operating frequency is at the resonance frequency. However, as the power generated is equal at the two optima, generated current is larger at short circuit conditions, whereas the voltage is larger under open circuit conditions.

The basic electromechanical model was extended to a 2-D cantilever beam model, including a modal analysis for a beam with a mass at the tip and the derivation of governing equations with an energy method. A preliminary model validation was completed, based on published measured results. The results were extended to a resonant analysis and power predictions made, resulting in a power density of $238 \mu \mathrm{W} / \mathrm{cm}^{3}$. The model was further extended to a piezoelectric energy harvester utilizing the longitudinal actuation mode and a micro-scale prototype device was analyzed. Frequency and tip displacement results compare well. The electrical performance of the device has not been predicted as the complete harvesting electronics have yet to be included.

A non-optimized preliminary design of a low-level MPVEH was performed and the performance was predicted for the next generation MPVEH device, which is to be built and tested. A power of $0.031 \mu \mathrm{W}$ from low-level vibrations was predicted, translating into a power density of $28.2 \mu \mathrm{W} / \mathrm{cm}^{3}$. With design optimization, it is likely that the power density will be increased by as much as an order of magnitude.

\section{ACKNOWLEDGMENTS}

The authors would like to gratefully acknowledge contributions from John Dugundji, Professor Emeritus, Department of Aeronautics and Astronautics, MIT, Cambridge, MA.

\section{REFERENCES}

1. http://robotics.eecs.berkeley.edu/ pister/SmartDust/, (2004).

2. http://www-mtl.mit.edu/research/icsystems/uamps/, (2004).

3. http://www.millennial.net/, (2004). 
4. E. Welsh, W. Fish, and J. P. Frantz, Proceedings of the 2003 IEEE International Symposium on Circuits and Systems, May 25-28, 2003, Bangkok, Thailand, (2003).

5. S. Roundy, P. K. Wright, and J. Rabaey, Computer Communications 26(11), 1131 (2003).

6. J. M. Rabaey, M. J. Ammer, J. L. J. da Silva, D. Patel, and S. Roundy, Computer 33(7), 42 (2000).

7. S. Meninger, J. O. Mur-Miranda, R. Amirtharajah, A. Chandrakasan, and J. Lang, Proceedings of the 1999 International Conference on Low Power Electronics and Design (ISLPED), Aug 16-Aug 17 1999, 48 (1999).

8. J. M. Rabaey, J. Ammer, T. Karalar, S. Li, B. Otis, M. Sheets, and T. Tuan, 2002 IEEE International Solid-State Circuits Conference, Feb 3-7, 2002, San Francisco, CA (2002).

9. R. Amirtharajah and A. P. Chandrakasan, IEEE Journal of Solid-State Circuits 33(5), 687 (1998).

10. A. Chandrakasan, R. Amirtharajah, J. Goodman, and W. Rabiner, IEEE International Symposium on Circuits and Systems, ISCAS, Monterey, CA, USA (1998).

11. S. Rhee, D. Seetharam, S. Liu, and N. Wang, UbiComp 2003, the Fifth International Conference on Ubiquitous Computing, Seattle, WA (2003).

12. I. Stark and M. Stordeur, 18th International Conference on Thermoelectrics (ICT'99), Aug 29-Sep 2, 1999, Baltimore, MD, USA (1999).

13. J. R. Lim, G. J. Snyder, C.-K. Huang, J. A. Herman, M. A. Ryan, and J.-P. Fleurial, Thermoelectrics, 2002. Proceedings ICT '02. Twenty-First International Conference on (2002).

14. G. J. Snyder, J. R. Lim, C.-K. Huang, and J.-P. Fleurial, Nature Materials 2(8), 528 (2003).

15. M. Strasser, R. Aigner, C. Lauterbach, T. F. Sturm, M. Franosch, and G. Wachutka, TRANSDUCERS, Solid-State Sensors, Actuators and Microsystems, 12th International Conference (2003).

16. P. Miao, A. S. Holmes, E. M. Yeatman, T. C. Green, and P. D. Mitcheson, Electrostatics, Edinborough, UK (2003).

17. P. D. Mitcheson, B. H. Stark, P. Miao, E. M. Yeatman, A. S. Holmes, and T. C. Green, Eurosensors, Portugal (2003).

18. T. Von Buren, P. Lukowicz, and G. Troster, Wearable Computers, Seventh IEEE International Symposium., White Plains, NY (2003).

19. T. Sterken, K. Baert, and S. B. Puers, Workshop on Semiconductor Sensors (SeSens), Veldhoven, Netherland (2002).

20. S. Roundy, P. K. Wright, and K. S. J. Pister, 2002 ASME International Mechanical Engineering Congress and Exposition, Nov 17-22 2002, New Orleans, LO, United States (2002).

21. K. O’Handley, Technical Report (2003).

22. C. B. Williams and R. B. Yates, Sensors and Actuators, A: Physical 52(1-3 pt 1), 8 (1996b). 
23. M. El-Hami, P. Glynne-Jones, N. M. White, M. Hill, S. Beeby, E. James, A. D. Brown, and J. N. Ross, EUROSENSORS, Copenhagen, Denmark (2000).

24. E. P. James, M. J. Tudor, S. P. Beeby, N. R. Harris, P. Glynne-Jones, J. N. Ross, and N. M. White, Proceedings Eurosensor XVI (2002).

25. J. M. H. Lee, S. C. L. Yuen, W. J. Li, and P. H. W. Leong, Proceedings of the 2003 IEEE International Symposium on Circuits and Systems, May 25-28 2003, Bangkok, Thailand (2003).

26. H. Kulah and K. Najafi, IEEE International Micro Electro Mechanical Systems (MEMS) Conference, Maastricht, The Netherlands (2004).

27. A. Mehra, X. Zhang, A. A. Ayon, I. A. Waitz, M. A. Schmidt, and C. M. Spadaccini, Journal of Microelectromechanical Systems 9 (4), 517 (2000).

28. A. S. Holmes, G. Hong, K. R. Pullen, and K. R. Buffard, 17th International IEEE Micro Electro Mechanical Conference, Maastricht, The Netherlands (2004).

29. http://www.panasonic.com, (2004).

30. H. A. Sodano, D. J. Inman, and G. Park, Shock and Vibration Digest 36(3), 197 (2004).

31. V. H. Schmidt, IEEE International Symposium on Applications of Ferroelectrics, Bethlehem, PA, USA (1986).

32. S. O. R. Moheimani, IEEE Transactions on Control Systems Technology 11(4), 482 (2003).

33. J. Onoda and K. Makihara, AIAA Journal 41(4), 711 (2003).

34. M. J. Konak, I. G. Powlesland, S. P. van der Velden, and S. C. Galea, Proceedings of the SPIE, Smart Structures and Materials 3241, 270 (1997).

35. N. G. Elvin, A. A. Elvin, and M. Spector, Smart Materials and Structures 10(2), 293 (2001).

36. H. A. Sodano, G. Park, and D. J. Inman, Strain 40(2), 49 (2004).

37. H. A. Sodano, G. Park, D. J. Leo, and D. J. Inman, 2003 ASME International Mechanical Engineering Congress, Washington, DC., United States (2003).

38. G. A. Lesieutre, G. K. Ottman, and H. F. Hofmann, Journal of Sound and Vibration 269(3-5), 991 (2004).

39. A. Kasyap, J. Lim, D. Johnson, S. Horowitz, T. Nishida, K. Ngo, M. Sheplak, and L. Cattafesta, Proceedings of 9th International Congress on Sound and Vibration, (Orlando, FL, 2002).

40. P. Glynne-Jones, S. P. Beeby, E. P. James, and N. M. White, Proceedings of 11th International Conference on Solid State Sensors and Actuators Transducers (Munich, Germany, 2001).

41. P. Glynne-Jones, S. P. Beeby, and N. M. White, IEE Proceedings-Science, Measurement and Technology 148(2), 68 (2001b).

42. N. M. White, P. Glynne-Jones, and S. P. Beeby, Smart Materials and Structures 10(4), 850 (2001). 
43. R. Duggirala, H. Li, and A. Lal, Solid-State Sensor, Actuator and Microsystem Workshop. (Hilton Head, South Carolina 2004).

44. M. J. Ramsay and W. W. Clark, Proceedings of the SPIE, Smart Structures and Materials 4332, 429 (2001).

45. S. Kim, W. W. Clark, and Q.-M. Wang, Proceedings of the SPIE, Smart Structures and Materials. San Diego, CA, USA (2003).

46. S. Kim, W. W. Clark, and Q.-M. Wang, ASME International Mechanical Engineering Congress. Washington, DC., United States (2003).

47. N. S. Shenck and J. A. Paradiso, IEEE Micro 21(3), 30 (2001).

48. J. Kymissis, C. Kendall, J. A. Paradiso, and N. Gershenfeld, Second IEEE International Symposium on wearable Computers. Pittsburg, PA (1998).

49. G. K. Ottman, H. F. Hofmann, A. C. Bhatt, and G. A. Lesieutre, IEEE Transactions on Power Electronics 17(5), 669 (2002).

50. G. K. Ottman, H. F. Hofmann, and G. A. Lesieutre, Proceedings of PESC 2002-Power Electronics Specialists Conference, 23-27 June 2002, Cairns, Qld., Australia (2003).

51. R. Sood, Y. B. Jeon, J.-H. Jeong, and S. G. Kim, Hilton Head 2004, Hilton Head, SC (2004).

52. W. N. Sharpe Jr. and J. Bagdahn, Mechanics of Materials 36(1-2), 3 (2004).

53. S. M. Spearing, Acta Materialia 48(1), 179 (2000).

54. C. B. Williams, R. C. Woods, and R. B. Yates, Proceedings of the IEE Colloquium on Compact Power Sources, May 8, 1996. London, UK (1996a).

55. H. Hosaka, K. Itao, and S. Kuroda, Proceedings of the IEEE Micro Electro Mechanical Systems, 193 (1994).

56. F. R. Blom, S. Bouwstra, M. Elwenspoek, and J. H. J. Fluitman, Journal of Vacuum Science \& Technology B: Microelectronics Processing and Phenomena 10(1), 19 (1992).

57. L. Meirovitch, Elements of Vibration Analysis, Second edition ed. McGraw-Hill, Inc., New York (1986).

58. J. O. Mur-Miranda, PhD, Massachusetts Institute of Technology (2004).

59. A. A. Bent, M. S, Massachusetts Institute of Technology (1994).

60. IEEE Standard on Piezoelectricity (IEEE, New York, 1987).

61. R. Sood, M. S., Massachusetts Institute of Technology (2003).

62. T. Ikeda, Fundamentals of Piezoelectricity. Oxford University Press, New York (1996).

63. H. A. Sodano, G. Park, D. J. Leo, and D. J. Inman, Proceedings of SPIE, Smart Structures and Materials. San Diego, CA, United States (2003).

64. J. Soderkvist, Journal of the Acoustical Society of America 90 (2, pt.1), 686 (1991).

65. N. W. Hagood, W. H. Chung, and A. Von Flotow, Journal of Intelligent Material Systems and Structures 1(3), 327 (1990).

66. D. J. Inman, Engineering Vibration. Prentice Hall, Upper Saddle River, NJ (1996).

67. C. W. S. To, Journal of Sound and Vibration 83(4), 445 (1982). 
68. D. C. D. Oguamanam, International Journal of Mechanical Sciences 45(6-7), 963 (2003).

69. B. Rama Bhat and H. Wagner, Journal of Sound and Vibration 45(2), 304 (1976).

70. N. Hagood, R. Kindel, K. Ghandi, and P. Gaudenzi, Proceedings of the SPIE, Smart Structures and Materials 1917(1), 341 (1993).

71. J. J. Bernstein, J. Bottari, K. Houston, G. Kirkos, R. Miller, B. Xu, Y. Ye, and L. E. Cross, 1999 IEEE Ultrasonics Symposium, Oct 17-Oct 20, 1999, Caesars Tahoe, NV USA (1999).

72. B. Xu, Y. Ye, L. E. Cross, J. J. Bernstein, and R. Miller, Applied Physics Letters 74(23), 3549 (1999).

73. L. J. Nelson, C. R. Bowen, R. Stevens, M. Cain, and M. Stewart, Proceedings of SPIE, Smart Structures and Materials, San Diego, CA, United States (2003).

74. W. Beckert and W. S. Kreher, 11th International Workshop on Computational Mechanics of Mat., Sep 24-25, 2001, Freiberg, Germany (2003). 